\title{
Basic Objects in Natural Categories
}

\author{
Eleanor Rosch, Carolyn B. Mervis, Wayne D. Gray, David M. \\ Johnson, and Penny Boyes-Braem \\ University of California, Berkeley
}

\begin{abstract}
Categorizations which humans make of the concrete world are not arbitrary but highly determined. In taxonomies of concrete objects, there is one level of abstraction at which the most basic category cuts are made. Basic categories are those which carry the most information, possess the highest category cue validity, and are, thus, the most differentiated from one another. The four experiments of Part I define basic objects by demonstrating that in taxonomies of common concrete nouns in English based on class inclusion, basic objects are the most inclusive categories whose members: (a) possess significant numbers of attributes in common, (b) have motor programs which are similar to one another, (c) have similar shapes, and (d) can be identified from averaged shapes of members of the class. The eight experiments of Part II explore implications of the structure of categories. Basic objects are shown to be the most inclusive categories for which a concrete image of the category as a whole can be formed, to be the first categorizations made during perception of the environment, to be the earliest categories sorted and earliest named by children, and to be the categories most codable, most coded, and most necessary in language.
\end{abstract}

The world consists of a virtually infinite number of discriminably different stimuli. One of the most basic functions of all organisms is the cutting up of the environment into classifications by which nonidentical stimuli can be treated as equivalent. Yet there has been little explicit attempt to determine the principles by which humans divide up the world in

This research was supported by grants to the first author (under her former name Eleanor Rosch Heider) by the National Science Foundation GB-38245X, by The Grant Foundation, and by the National Institutes of Mental Health 1 R01 MH24316-01. Portions of these data were presented in papers delivered at the meeting of the Psychonomic Society, Boston, November 1974 and at the meeting of the Society for Research in Child Development, Denver, April, 1975. We thank R. Scott Miller, Joseph Romeo, Ross Midgley, Clodio Norega, Meriska Huynen, James McLaughlin, Steven Mervis, John Schutz, Buzz Rigsby, Eugene Sanders, Steve Frank, and Denis Fridkis for help in performing the experiments. Our thanks to Oscar Anderson for contributions to the idea of using shape overlap as a measure of similarity and to Carol Simpson for programming the overlap measure. We are grateful to the students and staff of the University of California nursery school and the Pacific Grove public schools for their kindly cooperation in the developmental studies. We are very grateful to Donald Norman for editorial comments.

Carolyn Mervis is now at the University of Illinois. She was a National Science Foundation predoctoral fellow during performance of the research.

Requests for reprints should be sent to Eleanor Rosch, Department of Psychology, University of California at Berkeley, Berkeley, CA 94720. 
the way that they do. On the contrary, it has been the tendency both in psychology and anthropology to treat that segmentation of the world as originally arbitrary and to focus on such matters as how categories, once given, are learned or the effects of having a label for some segment. A typical statement of such a position is: ". . . the physical and social environment of a young child is perceived as a continuum. It does not contain any intrinsically separate 'things.' The child, in due course, is taught to impose upon this environment a kind of discriminating grid which serves to distinguish the world as being composed of a large number of separate things, each labeled with a name" (Leach, 1964, p. 34). It is the contention of the present paper that such a view would be reasonable only if the world were entirely unstructured; that is, using Garner's (1974) definition of structure, if the world formed a set of stimuli in which all possible stimulus attributes occurred with equal probability combined with all other possible attributes.

\section{PRINCIPLES OF CATEGORIZATION}

The aim of the present research is to show that the world does contain "intrinsically separate things." The world is structured because real-world attributes do not occur independently of each other. Creatures with feathers are more likely also to have wings than creatures with fur, and objects with the visual appearance of chairs are more likely to have functional sit-on-ableness than objects with the appearance of cats. That is, combinations of attributes of real objects do not occur uniformly. Some pairs, triples, or ntuples are quite probable, appearing in combination sometimes with one, sometimes another attribute; others are rare; others logically cannot or empirically do not occur.

By category we mean a number of objects which are considered equivalent. Categories are generally designated by names, e.g. dog, animal. A taxonomy is a system by which categories are related to another by means of class inclusion. The greater the inclusiveness of a category within a taxonomy, the higher the level of abstraction. Each category within a taxonomy is entirely included within one other category (unless it is the highest level category) but is not exhaustive of that more inclusive category (see Kay, 1971). Thus, the term level of abstraction within a taxonomy refers to a particular level of inclusiveness. A familiar taxonomy is the Linnean system for the classification of animals.

We will argue that categories within taxonomies of concrete objects are structured such that there is generally one level of abstraction at which the most basic category cuts can be made. In general, the basic level of abstraction in a taxonomy is the level at which categories carry the most information, possess the highest cue validity, and are, thus, the most differentiated from one another. The basic level of abstraction can be de- 
scribed both in general terms of cognitive economy and in the specific language of probabilistic cue validity (Brunswik, 1956).

\section{Cognitive Economy}

To categorize a stimulus means to consider it, for purposes of that categorization, not only equivalent to other stimuli in the same category but also different from stimuli not in that category. On the one hand, it would appear to the organism's advantage to have as many properties as possible predictable from knowing any one property (which, for humans, includes the important property of the category name), a principle which would lead to formation of large numbers of categories with the finest possible discriminations between categories. On the other hand, one purpose of categorization is to reduce the infinite differences among stimuli to behaviorally and cognitively usable proportions. It is to the organism's advantage not to differentiate one stimulus from others when that differentiation is irrelevant for the purposes at hand. The basic level of classification, the primary level at which cuts are made in the environment, appears to result from the combination of these two principles; the basic categorization is the most general and inclusive level at which categories can delineate real-world correlational structures.

\section{Cue Validity}

Cue validity is a probabilistic concept; the validity of a given cue $x$ as a predictor of a given category $y$ (the conditional probability of $y(x)$ increases as the frequency with which cue $x$ is associated with category $y$ increases and decreases as the frequency with which cue $x$ is associated with categories other than $y$ increases. [The precise mathematical form used to compute the conditional probability $y / x$ has varied (Beach 1964a, 1964b; Reed, 1972).] The power of cue validity in formation of the internal structure of categories has been demonstrated for both natural and for controlled artificial categories (Rosch \& Mervis, 1975). The cue validity of an entire category may be defined as the summation of the cue validities for that category of each of the attributes of the category. (Note that category cue validity is not a probability: (a) its value may exceed 1, (b) it does not have the same set theoretic properties as a probability. ${ }^{1}$

A category with a high total cue validity is, by definition, more differentiated from other categories than one of lower total cue validity. A

\footnotetext{
${ }^{1}$ Were category cue validity a true probability, the most inclusive category would always have the highest validity. This follows from the fact that if category $\mathrm{A}$ includes category $\mathrm{B}$, the probability that object $x$ belongs to category $\mathrm{A}$ always exceeds the probability at $x$ belongs to category B. Category cue validity refers to a psychological factor-the extent to which cues to category membership are available at all (attributes common to the
} 
working assumption of the present research is that in the real world information-rich bundles of perceptual and functional attributes occur that form natural discontinuities and that basic cuts in categorization are made at these discontinuities. Suppose that basic objects (e.g., chair, car) are at the most inclusive level at which there are attributes common to all or most members of the category. Then total cue validities are maximized at that level of abstraction at which basic objects are categorized. That is, categories one level more abstract will be superordinate categories (e.g., furniture, vehicle) whose members share only a few attributes among each other. Categories below the basic level will be subordinate categories (e.g. kitchen chair, sports car) which are also bundles of predictable attributes and functions, but contain many attributes which overlap with other categories (for example, kitchen chair shares most of its attributes with other kinds of chairs).

Superordinate categories have lower total cue validity than do basic level categories because they have fewer common attributes. Subordinate categories have lower total cue validity than do basic because they also share most attributes with contrasting subordinate categories. That basic objects are categories at the level of abstraction which maximizes cue validity is another way of asserting that basic objects are the categories which best mirror the correlational structure of the environment.

This paper has two major parts. Part I reports a series of converging experiments whose purpose was to define further and to provide evidence supporting the concept of basic level objects. Part II explores some of the implications of the concept of basic objects for cognitive representations of categories, perception, and the development of classifications, as well as for the linguistic usage and evolution of category labels.

\section{PART I: CONVERGING OPERATIONAL DEFINITIONS OF BASIC OBJECTS}

Basic objects are the most inclusive categories which delineate the correlational structure of the environment. However, real-world correlational structure itself provides a number of aspects which could serve as the point of departure for the present analysis. Four such aspects are explored in our experiments.

\section{Outline of Experiments 1 through 4}

Experiment 1 . Some ethnobiologists have asserted a claim somewhat similar to that of the present study-i.e., there is a level of biological 
classification which corresponds to "natural groupings" of organisms which possess "bundles" of correlated features and which are "obviously" different from other organisms (Berlin, 1972; Bulmer, 1967; Bulmer \& Tyler, 1968; Rosaldo, 1972). Berlin (1972) has also argued for an evolutionary theory of plant names. Berlin has identified natural grouping at the level of the genus (oak, maple) and has amassed considerable evidence in support of the claim that the first plant names to evolve in a language refer to this level. For our purposes, the work of ethnobiologists is limited by three factors. First, it refers only to biological classes; second, the claims for natural groupings are generally supported only by the ethnographer's mention of a few correlated attributes (a tendency being corrected in Berlin's current ethnobotanical studies - see Berlin, Breedlove, \& Raven, 1966); and finally, the location of natural groupings at a particular level of abstraction is defined by linguistic-taxonomic, rather than psychological, criteria (see Berlin, Breedlove, \& Raven, 1973). The purpose of the first experiment in this paper is to provide a systematic empirical study of the co-occurrence of attributes in the most common taxonomies of man-made and biological objects in our own culture.

Experiment 2. Among the attributes of objects are the ways in which humans habitually use or interact with them. For example, when performing the action of sitting down on a chair, a sequence of body and muscle movements are typically made which are aspects of the functions of chairs. It is not important here to determine whether material attributes or action sequences are more fundamental (although Piaget, 1952, has argued for the ontogenetic significance of sensori-motor schemata in concept development). Our claim is that groups of objects in a given culture require highly similar motor patterns in their use, and these motor patterns serve as common attributes in the construction of categories.

The purpose of Experiment 2 is to develop systematic techniques to operationalize and specify in some detail the actual motor programs which adults employ when using or interacting with common objects. The hypothesis is that when motor programs are measured, basic level objects will be the most inclusive categories at which consistent motor programs are employed for all objects of a class. Categories subordinate to the basic level should be characterized by essentially the same attributes and motor programs as the basic level.

Experiment 3. An aspect of the meaning of a category of objects, inseparable from the attributes and motoric uses of the objects, is the way the objects "look." Appearance is, perhaps, the most difficult of all characteristics of objects to define. Shape is a very general and important aspect of objects. Included within what we call shape are the structural relationships of the parts of an object to each other-for example, the visual representation of the legs, seat, and back of a chair and of the way 
in which those parts of the chair are placed in relation to one another. Hence, shape is probably a salient visual cue in normal recognition of objects. Pilot studies showed that objects are quite recognizable from only the outline tracing of a two-dimensional projection of their shapes. Indeed, objects were as recognizable from such outlines as from lists of criterial attributes or from full views of parts of the objects.

In order to measure similarity of shape between two objects, we normalized outline drawings of the objects for size and orientation, juxtaposed them, and computed the ratio of overlapped to nonoverlapped area. Experiment 3 examines the hypothesis that with this measure of similarity of shape, the basic level of categorization is the most inclusive level at which the objects of a class begin to look very much alike.

Experiment 4. If the basic level is the most inclusive level at which shapes of objects of a category are similar, the basic level might also be the most inclusive level at which an average shape of an object can be recognized. Thus, the basic level may be the most inclusive level at which it is possible to form a mental image of some "average" member of the class. This would be the most abstract level at which it is possible to have a relatively concrete image.

\section{EXPERIMENT 1: ATTRIBUTES}

Experiment 1 has three parts. First, the major source of data was obtained by asking subjects to list attributes of object names derived from nine taxonomies. Second, an independent group of subjects judged the truth of the attributes which had been most frequently listed by the first group of subjects. Third, subjects listed attributes of visually present objects as a check on the nature and validity of the attribute lists obtained when object names were used in the first part of the experiment.

\section{Methods}

\section{Attribute Listing}

Subjects. Subjects were 200 students in undergraduate psychology courses.

Stimuli. The stimuli were 90 object names belonging to three levels of abstraction in nine taxonomies (Table 1). These stimuli were chosen systematically according to two principles: One, as the study was an attempt to investigate the categorization of realworld material objects, the stimuli should represent the most common categories of concrete objects in our culture; two, as all of the hypotheses concerned levels of abstraction in classification of objects, specific stimuli had to be those for which relationships of class inclusion, superordination, and subordination both existed as potentials in the English language and were reliably agreed upon by normal speakers of English.

A measure of the most common superordinate categories of concrete objects was obtained in the following manner: The population of categories of concrete nouns in common use in English was determined by the concrete nouns with a word frequency of 10 
TABLE 1

The Nine Taxonomies USed as Stimuli

\begin{tabular}{|c|c|c|c|}
\hline Superordinate & Basic level & & linates \\
\hline \multicolumn{4}{|c|}{ Nonbiological taxonomies } \\
\hline \multirow{3}{*}{$\begin{array}{l}\text { Musical } \\
\text { instrument }\end{array}$} & Guitar & Folk guitar & Classical guitar \\
\hline & Piano & Grand piano & Upright piano \\
\hline & Drum & Kettle drum & Base drum \\
\hline \multirow[t]{3}{*}{ Fruit $^{\prime \prime}$} & Apple & Delicious apple & Mackintosh apple \\
\hline & Peach & Freestone peach & Cling peach \\
\hline & Grapes & Concord grapes & Green seedless grapes \\
\hline \multirow[t]{3}{*}{ Tool } & Hammer & Ball-peen hammer & Claw hammer \\
\hline & Saw & Hack hand saw & Cross-cutting hand saw \\
\hline & Screwdriver & Phillips screwdriver & Regular screwdriver \\
\hline \multirow[t]{3}{*}{ Clothing } & Pants & Levis & Double knit pants \\
\hline & Socks & Knee socks & Ankle socks \\
\hline & Shirt & Dress shirt & Knit shirt \\
\hline \multirow[t]{3}{*}{ Furniture } & Table & Kitchen table & Dining room table \\
\hline & Lamp & Floor lamp & Desk lamp \\
\hline & Chair & Kitchen chair & Living room chair \\
\hline \multirow[t]{3}{*}{ Vehicle } & Car & Sports car & Four door sedan car \\
\hline & Bus & City bus & Cross country bus \\
\hline & Truck & Pick up truck & Tractor-trailer truck \\
\hline \multicolumn{4}{|c|}{ Biological taxonomies } \\
\hline \multirow[t]{3}{*}{ Tree } & Maple & Silver maple & Sugar maple \\
\hline & Birch & River birch & White birch \\
\hline & Oak & White oak & Red oak \\
\hline \multirow[t]{3}{*}{ Fish } & Bass & Sea bass & Striped bass \\
\hline & Trout & Rainbow trout & Steelhead trout \\
\hline & Salmon & Blueback salmon & Chinook salmon \\
\hline \multirow[t]{3}{*}{ Bird } & Cardinal & Easter cardinal & Grey tailed cardinal \\
\hline & Eagle & Bald eagle & Golden eagle \\
\hline & Sparrow & Song sparrow & Field sparrow \\
\hline
\end{tabular}

"Fruit is not considered a biological taxonomy by the criteria in Berlin (1972).

or greater from the Kucera and Francis (1967) sample of written English. A superordinate category was considered in common use if at least four of its members met this criterion. Categories were eliminated if: (a) all of the items bore a part-whole relationship to the only reasonable superordinate (e.g., parts of the body, parts of buildings), (b) if there was linguistic ambiguity amongst possible superordinates (e.g., animal is commonly used as a synonym for mammal), and (c) if the superordinate cross-cut a large number of other taxonomic structures (e.g., food).

By these criteria, only one biological category, bird, could be included in the study. Because biological taxonomies were the only ones in which hypotheses concerning basic objects based on independent linguistic evolutionary data existed, it was necessary to amend the inclusion criteria. A biological category was included if at least one member of the category (or the superordinate noun itself) achieved a Kučera and Francis frequency 
of 10 or more. Thirteen superordinate categories met the new criteria: musical instrument, fruit, tool, clothing, furniture, vehicle, vegetable, toy, weapon, bird, tree, fish, and snake.

The second criterion for choosing items was the item's "taxonomic depth" in common English. To test our hypothesis, a class inclusion hierarchy with at least three levels of abstraction is needed: a basic level (such as hammer), a superordinate (such as tool), and subordinates into which the basic level can be further subdivided (such as claw hammer and ball-peen hammer). In order to determine taxonomic depth in common English for our potential items, the following procedure was followed: For each of the 13 categories, lists were constructed of the items in that category which appeared 10 or more times in the Battig and Montague (1969) tabulations. (These are tabulations of the frequencies with which instances were produced in response to the category name.) Five judges were given the lists. Judges were asked to give the superordinate (or superordinates if they felt more than one was appropriate) for each item and to list any or all subordinates of the item (i.e., any classifications into which the item could be further subdivided).

The final nine taxonomies used in the experiment each included at least three basic level objects which met the following criteria: (a) All five judges agreed unanimously on listing that superordinate as the single superordinate for the item, and (b) For each basic level object, there were at least two common subordinate items listed by all five judges. In addition, for biological taxonomies, we checked the official taxonomic legitimacy of listed subordinates before including them.

Procedure. The stimuli were divided into 10 sets of nine items each. Each set consisted of items at only one level of abstraction. Thus, there was one set of superordinate items, three sets of basic level items, and six sets of subordinate level items. For the basic level items, there was never more than one item per set which belonged to a given superordinate category, and for the subordinate items each set contained only one item from a given basic level (which entailed also from a given superordinate) category.

Subjects were tested in groups, and 20 different subjects received each of the 10 sets. Each item was printed at the top of a page and the nine pages of items in a set assembled in a different randomized order for each subject. Subjects were given $1 \frac{1}{2} \mathrm{~min}$ per item to write down all of the attributes of each object which they could think of. They were asked to avoid simple free associations. (For the complete text of the instructions, see Rosch, Mervis, Gray, Johnson. \& Boyes-Braem, Note 1.)

An initial tally was made, and only attributes which were listed by at least six subjects were used as data in the final tabulation.

\section{Judgments of Attributes}

A new group of subjects was asked to judge the truth of the attributes which had been listed by six or more subjects in the free listing experiment; they were not asked to introduce attributes themselves.

Subjects. Subjects were students in a seminar. Seven completed and returned the judgment forms out of interest in the experiment.

Stimuli. Separate forms for each of the 27 basic level items were rated by each subject. All of the attributes which had been listed six or more times for a basic level item, its superordinate, or its subordinates in the previous part of the experiment were listed, in random order, down the left side of the page. Across the top of the page were written the superordinate category name, the basic level name, and the two subordinate level names.

Procedure. Potential subjects were given forms for all 27 basic level items (which were arranged in different random order for cach subject) and asked to judge whether each of the attributes listed down the side of the page was true of each category listed across the top. (The text of the instructions is available in Rosch et al.. Note 1.) Subjects 
took the forms home with them; completed forms were collected at the regular seminar meeting the following week.

\section{Attributes of Physically Present Objects}

Both the free listing of attributes and the judgment of attributes in the previous parts of this experiment relied on subjects' retrieval from memory of object characteristics from their names. A further question was whether attribute lists produced by a subject listing the attributes of 20 different visually present objects of a category would correspond with the lists obtained from the previous 20 subjects listing attributes from memory for the object name.

Two paid subjects were employed for the task. Stimulus sets consisted of one basic level item from each of the nine taxonomies: guitar, apple, hammer, pants, chair, car, tree, fish, and bird (the use of the supposed superordinates for the biological taxonomies will be explained in the results section). The subjects' task was to find 20 different examples of each of the items listed above, and (timing themselves with a stopwatch) to list as many attributes of each item as possible in $1 \frac{1}{2} \mathrm{~min}$. Subjects were told to start with the objects in their own homes when applicable (such as chair and pants) and then to find the other items in the appropriate places: supermarkets, hardware stores, the street, or pictures in books. For fish, one subject visited the Steinhart Aquarium at Golden Gate Park, one rated pictures in the Crescent Book series Color Treasury of Aquarium Fish (1972). For birds, both subjects used both Birds of North America: A Guide to Field Identification (1966) and The Golden Book of Bird Stamps (1966), each picking 20 different pictures from the books using tables of random numbers. Subjects were given essentially the same instructions for listing attributes as the first group of subjects except that they were told not to list functional attributes (which they found themselves simply repeating without further consideration for each instance of the object once they had thought of the attribute).

The protocols for each subject were tallied separately in the same manner as had been the protocols for the 20 subjects listing attributes for each object name in the initial free listing condition.

\section{Results and Discussion}

Table 2 shows the number of attributes listed six or more times for each level of abstraction. The left-hand columns show the tallies obtained from the attribute listings in the first part of the experiment. The righthand columns show the judge-amended tallies; in computing these tallies, an attribute was added or subtracted only when all seven judges agreed that it belonged or did not belong at the appropriate level. Appendix 1 shows examples of the judge-amended attributes: a list of all of the judgeamended attributes for each of the nine taxonomies is available in Rosch et al., Note 1.

The basic hypothesis of the study was that the basic level would be the most inclusive level in a taxonomy at which a cluster of attributes, believed to be common to the class named, would be listed. An initial glance at Table 2 shows that the results for the nonbiological and the biological taxonomies are quite different-that is, what we had taken to be the superordinate level for the biological items showed all the signs of being basic level objects at least for our subjects. The first test of significance 
TABLE 2

Number of Attributes in Common at Each Level of Abstraction

\begin{tabular}{|c|c|c|c|c|c|c|}
\hline \multirow[b]{3}{*}{ Category } & \multicolumn{6}{|c|}{ Number of attributes in common } \\
\hline & \multicolumn{3}{|c|}{ Raw tallies } & \multicolumn{3}{|c|}{ Judge-amended tallies } \\
\hline & $\begin{array}{c}\text { Super- } \\
\text { ordinate }\end{array}$ & $\begin{array}{l}\text { Basic } \\
\text { level }\end{array}$ & $\begin{array}{c}\text { Sub- } \\
\text { ordinate }\end{array}$ & $\begin{array}{c}\text { Super- } \\
\text { ordinate }\end{array}$ & $\begin{array}{l}\text { Basic } \\
\text { level }\end{array}$ & $\begin{array}{c}\text { Sub- } \\
\text { ordinate }\end{array}$ \\
\hline \multicolumn{7}{|c|}{ Nonbiological taxonomies } \\
\hline \multicolumn{7}{|l|}{ Musical } \\
\hline Fruit & 7 & 12.3 & 14.7 & 3 & 8.3 & 9.5 \\
\hline Tool & 3 & 8.3 & 9.7 & 3 & 8.7 & 9.2 \\
\hline Clothing & 3 & 10.0 & 12.0 & 2 & 8.3 & 9.7 \\
\hline Furniture & 3 & 9.0 & 10.3 & 0 & 7.0 & 7.8 \\
\hline Vehicle & 4 & 8.7 & 11.2 & 1 & 11.7 & 16.8 \\
\hline \multicolumn{7}{|c|}{ Biological taxonomies } \\
\hline Tree & 9 & 10.3 & 11.2 & 10 & 11.0 & 11.5 \\
\hline Fish & 6 & 8.7 & 9.3 & 8 & 9.7 & 10.0 \\
\hline Bird & [1] & 14.7 & 15.3 & 14 & 16.0 & 16.5 \\
\hline
\end{tabular}

was, therefore, a test of whether results for the biological and nonbiological categories (e.g., the level at which a marked increase in attributes occurred) differed significantly; such a test was necessary before the substantive hypothesis of the study could be tested. For each category, the proportion of basic level attributes contributed by the increase in attributes between the hypothesized superordinate and the hypothesized basic level was computed. The difference in that proportion between the nonbiological and the biological categories was computed separately for the raw and the judge-amended tallies. Both proved significant: raw tallies, $t(7)=3.72, p<.01$; judge-amended tallies, $t(7)=4.11, p<.01$. This finding supports the idea that for our subjects, the hypothesized superordinate appeared to be the basic level for the biological taxonomies.

Tests of the difference in number of attributes between superordinate and basic level categories were performed for the nonbiological taxonomies to test the hypothesis that basic level items would contain more attributes than superordinate level items. The tests were significant: raw tallies, $t(5)=4.75, p<.01$; judge-amended tallies, $t(5)=10.07$, $p<.001$. The second prediction from the substantive hypothesis, that the number of attributes added at the subordinate level would be significantly fewer than the number added at the basic level, was also supported for the nonbiological taxonomies: raw tallies, $t(5)=4.28$, 
$p<.01$; judge-amended tallies, $t(5)=6.43, p<.001$. (Neither hypothesis could be tested for the biological taxonomies since we had not obtained attribute lists for levels of classification superordinate to the hypothesized superordinates.) Thus, for those categories in which the location of basic level objects is clear, the basic level does appear to be the most inclusive level at which objects have clusters of attributes in common.

In an additional analysis, attributes were coded separately as nouns, adjectives, or functional attributes. The majority (seven out of 10) of the total number of attributes listed for the superordinates of the nonbiological taxonomies were functional attributes of a very general nature. The percentage of gain in function, noun, and adjective attributes for basic level over superordinate and for subordinate over basic level was tested for significance by the Sign Test. Both nouns and adjectives increased significantly more than functional attributes between the superordinate and basic level. For the subordinate level, however, those few attributes which were added were almost exclusively adjectives rather than nouns (Sign test, $p<.05$ ).

The foregoing analysis was based upon attributes elicited by category names. In the last part of the experiment, attributes elicited by objects were compared with those obtained using object names. (Because this part of the experiment was undertaken after the name elicited analyses had been completed, tree, fish, and bird were treated as basic level objects rather than superordinates.)

The attributes listed by the two subjects were tallied in the same manner as has been described for the previous data. Reliability between the two subjects was high; exactly the same attributes were and were not listed six or more times by both subjects in all but one instance.

Results of these tallies were clear-cut. Attributes listed six or more times by the previous 20 subjects responding to one object name and attributes listed by two subjects six or more times to 20 instances of the object were in virtually perfect agreement. For all of the nine categories, every attribute listed in the judge-amended tallies was also listed by both subjects describing concrete objects. Furthermore, few (an average of only one per item) additional attributes were listed. (Added attributes are itemized in Rosch et al., Note 1.)

In summary: The hypothesis that there is a basic level of abstraction in taxonomies of common objects which is the most inclusive level at which the objects of a category possess numbers of attributes in common was supported for attribute listings obtained by three different methods: free listing of attributes for object names, judge-amended tallies of attributes for object names, and free listing of attributes for visually present objects. The one unexpected finding was that, for biological taxonomies, the basic level appeared to be the next higher level in the taxonomy than had 
been initially proposed by anthropological and linguistic-taxonomic evidence.

An important issue is the extent to which these findings represent descriptions of the correlational structure of attributes which are located in the real world versus in the knowledge and viewpoint of the subjects. That listing of attributes for visually present objects replicates the findings from attributes listed from memory argues that the findings are not an artifact of the way in which object names are stored in memory. However, the degree of expertise of subjects did not vary systematically (see Rosch et al., Note 1), and it is not known to what extent that may have affected location of the basic level, particularly for the biological taxonomies. Would, for example, an ichthiologist, whether presented with an actual example of a category or with a fish name, have been able to list sufficicnt attributes spccific to trout, bass, and salmon that the basic level for fish would have been placed at that level of abstraction? The issue of differential knowledge of correlational structures and the issue of the placement of basic level objects in biological taxonomies will be discussed further after findings of the other experiments have been described.

\section{EXPERIMENT 2: MOTOR MOVEMENTS}

The major hypothesis of this study was that basic level objects are the most inclusive categories for which highly similar sequences of motor movements are made to objects of the class. Testing the hypothesis required: first, obtaining a corpus of subjects' descriptions of the body and muscle movements which they made in interaction with objects (the level of abstraction of the object names being varied systematically as in Experiment 1); second, the development of a reliable coding system for those sets of descriptions of muscle movements; and, third, a tally of the numbers of motor movements in common for the category terms at the various levels of abstraction.

The major data derive from protocols produced by subjects asked to describe their body and muscle movements when interacting with specified objects. As a check on the validity of the descriptive protocols, naïve models performed the main general activity which had been elicited by the object name with a sample of actual objects (e.g., a model sat down in a chair), and subjects described the actual body and muscle movements which they observed.

\section{Methods}

\section{Descriptions of Imagined Movements to Object Names}

Subjects. Subjects were volunteers from psychology classes who received course credit for participation. Protocols were not used if the subject was not a native English 
speaker, did not complete the test, or clearly and obviously failed to follow instructions for all nine of the items. Two hundred protocols were obtained which met our criteria.

Stimuli. Stimuli were the same nine taxonomies used in Experiment 1 (Table 1), again divided into 10 sets of nine words. Twenty subjects received each set.

Procedures. As in Experiment 1, subjects were tested in groups. The instructions were preceded by a pantomime demonstration by the experimenter of bicycle riding, and by practice in naming movements. The experimenter solicited movement names for the different parts of the body from the subjects, getting every subject to utter at least one answer to a motor movement of a body part. The instructions asked the subjects to "write down the muscle movements that you make when you use or interact with that object, in as much detail as you can." (The text of the instructions is available in Rosch et al., Note 1.)

The name of each item was printed at the top of a blank page. At the end of $3 \mathrm{~min}$, subjects were asked to stop, turn the page, and proceed with the next item. With the exception of some of the first subjects tested, each subject who received the same set of nine items received them in a different random order.

\section{Descriptions of Actual Movements of Naïve Models}

Subjects were a class of 28 students. Four of the students were chosen at random to be the models. They were asked to wait outside the room while instructions were given to the remainder of the class; thus, they did not know the purpose of the experiment at the time that they performed their actions.

The class was told that previous subjects had been asked to describe the body and muscle movements which they imagined themselves making to objects, but that they would be asked to describe the actual body and muscle movements which the models would make when they interacted with actual objects. In order to give this group the same explanation of what was meant by specific body and muscle movements as had been given the previous subjects, the identical instructions were read to them, including their participation in the pantomimed bicycle riding. In addition, the subjects were told to watch the model carefully and to write down the body and muscle movements he or she had made in interacting with the object in as much detail as possible.

The models were called in, one at a time, and each performed with one object. The four activities were: sitting down on a chair, eating a small bunch of grapes, putting on a sock, and hammering a nail. The class was given 3 min following the completion of each modeled action to write down the sequence of movements.

Of the 24 protocols collected, two were eliminated because the subjects were not native English speakers, and two were eliminated at random for computational purposes.

\section{Results}

\section{Coding of Responses}

Considerable effort was required to develop a coding system for reducing the data which was both reliable and yielded similarity scores for units of movement which offered a face valid embodiment of the meaning of our hypothesis concerning similarity of movements.

The steps for coding the protocols were as follows: Each subject's protocol was first divided into the major activities for which that subject described an interaction with that object. For example, while the activity 
described for chairs was usually the process of sitting down on one, some subjects also described a muscle movement sequence for picking one up and carrying it, and other idiosyncratic responses. A finer coding was then applied separately to each major activity described by each subject. At each point in the description, the activity was segmented into the body part involved (head, eyes, neck, torso, arms, legs, knees, feet, hands, fingers, wrist, etc.). Under the body part, each activity (such as bending, rotating, turning, extending, etc.) described was listed. If the same body part was mentioned again later in the description with the same or a different movement, it was coded again with its new movement. (That movement sequences were not simply analytic descriptions of the meaning of the name of the major activity was demonstrated by the fact that similarly named activities, such as sitting or looking, received quite different movement lists when applied to different objects - e.g., sitting on a chair versus sitting as part of entering a car; looking at a bird versus looking at a tree.) Reliabilities (percentage of rater agreement) for the coding of nine randomly chosen protocols, each for a different object, three at each of the three levels of abstraction, were all $88 \%$ or higher for three independent coders.

The result of primary interest was the degree of similarity between movements made to objects at the three different levels of abstraction. The same movement was considered to have been included in the protocols of two different subjects only if, for the same basic activity in relation to an object, the same movement of the same body part occurred in the same place in the same sequence of movements. For example, if two different subjects mentioned bending their knees as part of the sitting process and that mention occurred between the actions of approaching and/or turning their back to the chair and the actions of touching their buttocks to and/or releasing their weight onto the chair, the bending of the knees was tallied as two occurrences of the same movement. However, if one subject mentioned bending his knees as part of raising his feet to rest them on a desk after having already put his weight onto the chair, it was coded as a different movement and not tallied with the other two bendings of knees. For motion sequences which, in fact, occur simultaneously (e.g., steering and food pedal manipulation while driving), sequence requirements for coding movements the same were adjusted to fit that fact. The criterion for counting a common movement was set at four or more responses.

The second count of body part movements was based on a far simpler measure. This tally was obtained to measure the degree of specificity of the descriptions at each level of abstraction regardless of the overlap in descriptions. To obtain this measure, the raters simply counted each specific movement rated, regardless of body part, place in the sequence, 
place in the major activity, or repetition of mention of the same movement by other subjects. This count was designed to examine the question of the specificity of the movements described for the class of objects named independently from the question of the similarity of movements made to objects of that class.

\section{Substantive Results}

Table 3 shows tallies of movements in common for the descriptions of imagined movements for the three levels of abstraction. Examples of some of the movements, listed under their appropriate major activity and in their appropriate sequence are shown in Appendix 2. (A complete listing of all movements for all items is available in Rosch et al., Note 1.) The proportion increase in number of attributes between the hypothesized superordinate and basic level categories differed significantly for the nonbiological and the biological categories: $t(7)=4.59, p<.01$. For the nonbiological taxonomies, significantly more motor movements were given in common for the basic level than for the superordinate, $t(5)=14.19$, $p<.001$. (As in Experiment 1, this effect could not be tested for the biological taxonomies alone.) The number of motor movements given in common for the subordinate level did not differ significantly from the

TABLE 3

Number of Motor Movements in Common at Each Level of Abstraction

\begin{tabular}{|c|c|c|c|c|c|}
\hline \multirow[b]{2}{*}{ Category } & \multicolumn{5}{|c|}{ Number of motor movements in common } \\
\hline & $\begin{array}{c}\text { Super- } \\
\text { ordinate }\end{array}$ & $\begin{array}{l}\text { Basic } \\
\text { level }\end{array}$ & $\begin{array}{l}\text { Subordinate } \\
\text { mean }\end{array}$ & $\begin{array}{c}\text { Subordinate } \\
\text { number } \\
\text { added }\end{array}$ & $\begin{array}{c}\text { Subordinate } \\
\text { number } \\
\text { subtracted }\end{array}$ \\
\hline \multicolumn{6}{|c|}{ Nonbiological taxonomies } \\
\hline $\begin{array}{l}\text { Musical } \\
\text { instrument }\end{array}$ & 0 & 16.7 & 16.2 & 2.2 & 2.6 \\
\hline Fruit & 4 & 21.3 & 20.5 & 2.5 & 3.3 \\
\hline Tool & 2 & 19.2 & 18.0 & 1.2 & 2.7 \\
\hline Clothing & 2 & 19.0 & 19.2 & 1.5 & 1.5 \\
\hline Furniture & 1 & 11.7 & 12.3 & 1.3 & .7 \\
\hline Vehicle & 1 & 18.0 & 18.2 & 2.8 & 2.5 \\
\hline \multicolumn{6}{|c|}{ Biological taxonomies } \\
\hline Tree & 8 & 6.0 & 6.8 & .7 & .8 \\
\hline Fish & 17 & 14.0 & 17.0 & 1.2 & 1.7 \\
\hline Bird & 7 & 7.3 & 7.2 & .3 & .5 \\
\hline
\end{tabular}


basic level: nonbiological taxonomies, $t(5)=.92$, ns.; biological taxonomies, $t(2)=1.83$, ns.

Because each of the motor movements tallied was an individual item in its unique place in a motor sequence, a tally of the movements to subordinate level objects which averaged movements added to and those omitted from the movement sequences tallied for the basic level object did not seem a totally sufficient test of the possible differences between movements described for basic level and for subordinate level objects. The two right-hand columns of Table 3, thus, show, respectively, the mean number of added and the mean number of omitted movements for subordinates in each taxonomy. The significance of the difference between the basic level and the subordinates was tested separately for the case in which only additions to the basic level tallies were counted for the subordinate and for the condition in which only omissions from the basic level tally were counted for the subordinate. None of those $t$-tests reached significance. Thus, even when additions and omissions are analyzed separately, there is no significant difference between the number of movements described in common for basic level and subordinate level categories of objects. The face validity of these results is apparent from the actual movement lists (Rosch et al., Note 1); the movements shown added to or excluded from the tallies of subordinate over basic level objects, in general, appear to be random fluctuations in the few movements which did or did not reach the criterion tally rather than movements specific to the subordinate object named.

The second measure of motor movements was a specificity count which included all movements listed regardless of whether or not they were listed in common. Table 4 shows the means for this tally. As in the count of movements in common, the proportion of increase in number of movements between the hypothesized superordinate and basic level categories differed significantly for the nonbiological and the biological taxonomies: $t(7)=5.18, p<.01$. For the nonbiological taxonomies, the specificity count was significantly greater for the basic level than for superordinate objects: $t(5)=11.93, p<.001$. Far from being more specific than the

TABLE 4

Specificity Count at Each Level of Abstraction

Mean number of movements

\begin{tabular}{lccc}
\cline { 2 - 4 } Category type & Superordinate & Basic level & Subordinate \\
\hline Nonbiological & 18.2 & 49.0 & 45.3 \\
Biological & 28.3 & 25.7 & 22.0 \\
\hline
\end{tabular}


basic level, the actual means for subordinate level objects for both the nonbiological and biological taxonomies were lower, on the general specificity count, than the means for the basic level objects, a difference which, however, did not reach significance: nonbiological taxonomies, $t(5)=2.41$, ns.; biological taxonomies, $t(2)=3.42$, ns.

These data on motor movements were obtained from subjects' descriptions of imagined movements with object names as stimuli; in the second part of the experiment, the same type of protocols were obtained by asking subjects to observe actual movements with real objects. As in the case of attribute listing, these descriptions varied remarkably little from the imagined descriptions. For three of the four actions performed, all of the movements obtained in the analysis of imagined action were also obtained from protocols based on observed actions. In each case, a small number of additional movements also achieved a frequency of four or greater for the observed actions - an average of 2.7 attributes added to an average of 19 original ones. All additions are listed in Rosch et al., Note 1. Additions were all finer differentiations or extensions of actions already specified in the original sequence. Thus, there is evidence that the protocols on which the conclusions are based, though obtained for imagined actions, would not have been substantially different were they all to have been obtained for observed actions.

In summary: The basic hypothesis of the study was supported both for number of common movements and total number of movements; superordinate categories have few, if any, motor movements that can be made to the category as a whole and few movements in common. Basic level categories receive descriptions of many specific movements made to all members of the category and many of these movements are described by a sufficient number of different subjects to form a picture of movement sequences made in common to all members of the basic level class of objects. Objects subordinate to the basic level did not differ significantly from the basic level either in the specificity of the descriptions or in the number of common movements made to the object. As in Experiment 1, the hypothesized superordinate level for the three biological categories showed all the characteristics of the basic level, and the hypothesized basic level for the biological taxonomies showed the characteristics of subordinates.

\section{EXPERIMENTS 3 AND 4: SHAPES OF OBJECTS}

To minimize the dependency on linguistic coding (a possible problem in the first two experiments), it is necessary to find a method for analyzing similarity in visual aspects of objects which is not dependent upon subjects' descriptions, which is free from effects of using object names, 
and which goes beyond similarity of the analyzable, listable attributes which have already been explored in Experiment 1.

The basic hypothesis of Experiments 3 and 4 is that shapes of objects show the same correlational structure as do attributes and motor movements. Experiment 3 tests whether the same basic objects identified in Experiments 1 and 2 would prove to be the most inclusive categories in which the shapes of objects would show a gain in objective similarity over the next higher level of abstraction. Experiment 4 tests whether these same basic level objects would be the most inclusive categories in which shapes are sufficiently similar to render the shape of an average of more than one member of the category identifiable as a category member. Before performing these experiments, we demonstrated that shape was a reasonable aspect of an object to use. Several pilot studies were performed (described in Rosch et al., Note 1) that demonstrated that objects were readily identified (at a rate greater than $90 \%$ ) from the two-dimensional outlines used in the experiments.

\section{Choice of stimuli}

Choice of the pictures of objects to be used in these experiments was an issue of particular importance. It is clear that the hypotheses of both experiments could easily be confirmed or disconfirmed by a biased choice of the pictures of objects representing each category. Thus, it was necessary to have an essentially random sample of pictures of objects for any category used: however, some of the nine taxonomies used in Experiments 1 and 2 did not lend themselves to such a choice procedure. On the basis of the availability of large numbers of pictures of members of the category, four hypothesized basic level categories from each of four superordinates (a total of 16 basic level categories) were chosen. These categories are shown in Table 5.

For each basic level category shown in Table 5, a pool of at least 100

TABLE 5

Classifications of Stimuli Used in Experiments 3 and 4

\begin{tabular}{cl}
\hline Superordinate & \multicolumn{1}{c}{ Basic level } \\
\hline Clothing & pants, shirt, shoes, socks \\
Vehicle & car, truck, airplane, motorcycle \\
Animals" & cat, dog, fish, butterny \\
Furniture & chair, table, sofa, bed \\
\hline
\end{tabular}

"For an objective measure of shape, the confusion between the terms animal and mammal in English is not relevant. 
pictures was obtained. More than half of the pictures in each category (and almost all of the animal pictures) were taken from books. The rest were obtained by photographing objects in the environment-vehicles on the streets and items of furniture and clothing in homes and in stores.

An additional sampling problem was presented by the orientation of the objects in the pictures. There are an infinite number of possible projections of a three-dimensional object in a two-dimensional representation. Rules were, therefore, required for defining and equating the orientations of objects. Fortunately, there is high agreement among subjects concerning the primary (and in many cases, secondary) canonical orientation in which objects are imagined. To obtain normative data, a class of 61 students was asked to list the orientation in which each of the basic level objects in Table 5 were imagined. There was high agreement (over $90 \%$ of the students) that all of the articles of furniture and two of the articles of ciothing (pants and shirts) were imagined primarily in a front view. The other two types of clothing (shoes and socks) were imagined from side as well as front views; however, all subjects agreed that they found imagining shoes and socks from a front view acceptable. For all of the furniture and clothing, front view pictures were, therefore, used. There was high agreement $(90 \%)$ that all of the vehicles were imagined in a side view. Side views predominated for animals, with some weight given to front views; besides all subjects whose primary orientation for an animal was front agreed that imagining the animals from the side was acceptable. Thus, side view pictures were used for the vehicles and animals. Since none of the hypotheses required comparing items between different superordinates, the difference in the side- and front-oriented categories did not present a problem. (The high agreement on canonical orientation is itself of interest; one may speculate that the canonical imagined orientation represents the most informative perspective in which to view the object by the same definitions of informativeness used in characterizing basic level objects in the present study.)

From the pool of 100 or more potential pictures, the four pictures to be used for each category were selected in two stages: In the first (nonrandom) stage, pictures were discarded on the basis of factors which would make them unusable in the study-for example, an object photographed in other than its canonical orientation or the picture of an object interrupted by another object in the foreground. No picture was discarded because of the shape of the object. In the second stage, the four pictures to be used werc selected randomly from the remaining pool.

This use of random choice of objects at the basic level had an effect on the availability of subordinate classes; pictures chosen were not always classifiable as a member of any particular subordinate class. To obtain subordinate classifications, four judges gave subordinate names for each 
of the 64 pictures. Six of the 16 basic level categories used proved divisable into two objects rated as members of one subordinate class with the other two pictures rated as members of a different subordinate class. These six basic level classes were: cars (sports car, sedan car); tables (kitchen table, dining room table); chairs (kitchen chair, living room chair), pants (dress pants, levis); shoes (women's shoes, tennis shoes); and socks (athletic socks, dress socks). Although these were only six out of 16 possible subordinate divisions, these appeared to be naturally occurring subordinates which had come out of a random choice of pictures of basic level objects and, as such, appeared to be of greater validity for testing the hypothesis than nonrandomly chosen subordinates for each basic level classification would have been.

Once the pictures were selected, it was necessary to normalize size and, when needed, to adjust orientation within the picture plane. This normalization took place in two stages. In the first stage, orientation was adjusted and size was approximately normalized photographically. That is, the objects in the photographs were enlarged, reduced and/or rotated to an approximately standard size and orientation by photographic means. At this stage, size was equated only in the sense that the pictures were reduced to the same order of magnitude; all now occupied a $8.89 \times 12.70$ $\mathrm{cm}$ framed space. The outlines of the 64 normalized pictured objects were then traced. These outline shapes were the raw material for the stimuli in the pilot studies and in Experiments 3 and 4. Examples of these outlines are shown in Fig. 1 in which one of the four objects in each basic level category is reproduced. In Fig. 1, the size of the outlines is reduced to approximately one-eighth of the original size. (To compute the ratio of overlapped areas in Experiment 3, the areas of the shapes were equated more precisely by computer.)

\section{EXPERIMENT 3}

\section{Methods}

\section{Stimuli and Procedures}

The categories used are shown in Table 5.

To compute the ratios of overlaps of the normalized shapes, tracings of the shapes were projected by an overhead projector onto a display oscilliscope. Tracings of the two pictures to be compared were overlapped, orientation remaining constant.

Computation of the overlap may be thought of in Venn Diagram terminology. Two juxtaposed shapes, $\mathrm{A}$ and $\mathrm{B}$, have an area in common, $\mathrm{AB}$, and areas not in common, $\mathrm{A}$ minus $\mathrm{AB}$ and $\mathrm{B}$ minus $\mathrm{AB}$. For present purposes, it was necessary to compute the ratio of $A B$ to [( $A$ minus $A B)$ plus $(B$ minus $A B)]$. To do this, the area covered by each shape was first traced with a light pen onto the projections on the display scope. A program computed the ratio of the second shape to the first and adjusted the area of the second by the factor necessary to equalize it to the area of the first. Following this normalization of area, the areas covered by either shape (the union) and by both shapes (the intersection) were traced. The program then computed the ratio of overlap. 


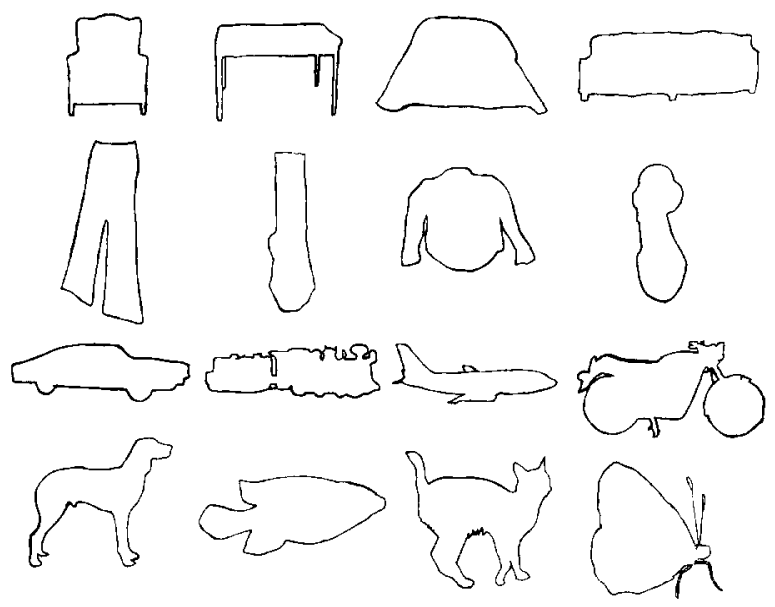

FIG. 1. Examples of traced outlines of pictures used in Experiment 3. One example of each basic level object from each superordinate category is shown.

Because it was not practical to compute ratios for all possible combinations of the 64 pictures taken four at a time, one of the four basic level object pictures chosen at random from each of the 16 groups of basic level object pictures was compared with each of the other three basic level object pictures in its group. This procedure produced a total of three comparisons within each basic level group of pictures. The mean of these three ratios was taken as a measure of the average overlap within that basic level category.

The same randomly chosen picture that had been compared with the other three pictures within a basic level group was also compared with one randomly chosen member of each of the other three basic level groups within the superordinate (see Table 5). The mean of these three comparisons was considered a measure of the ratio of overlap for that basic level group with other members of the superordinate category. For example, if shirt 2 were chosen to represent shirts, it was compared with shirts 1,3 , and 4 , and was then compared with one randomly chosen member of each of the other basic level items of clothing-i.e., with one randomly chosen shoe, one sock, and one pair of pants. The means of the first three and second three comparisons were taken as the data points from which to compare the ratio of overlap among shirts with the ratio of overlap between shirts and other clothing.

For comparison of the within subordinate overlap ratios with the within basic level overlap ratios a very similar procedure was followed. Each of the four items in a basic level group containing two subordinates was compared with each of the others, and each item was compared with one randomly chosen item from each of the other basic groups within its superordinate. From these comparisons, 27 mean overlap scores, three for each of the subordinate item pairs, were obtained. The three scores to be compared for each of the six items were: (a) the mean ratio of overlap between the two pairs of subordinate item, (b) the mean ratio of overlap between each of those same items with the other two items, not of the same subordinate, within the same basic level group, and (c) the mean overlap of those same two items with each of the items in the same superordinate class.

\section{Results}

Of the 16 pairs of comparisons between the mean overlap ratios of items within the same basic level category with items within the same super- 
ordinate category, 15 were in the direction within-basic-level ratios greater than within-superordinate-level ratios. The one reversal in sign was the smallest difference of the 16 . Such results are highly significant by the Wilcoxon Matched-Pairs Signed-Ranks test, $p<.001$. Of the six comparisons of subordinate with basic level overlaps, five showed greater overlap within subordinate categories than within basic level (a difference which reached significance by the $t$ test: $t(5)=2.91, p<.05$ ); however, in all six cases, the difference between increase in overlap which was gained by comparing subordinates with each other over comparing basic level objects with each other was far less than the increase in overlap gained by comparing members of the same superordinate with each other: Wilcoxon, $p<.05 ; t(5)=7.31, p<.001$.

In summary, the basic hypothesis is supported. A large and consistent increase in similarity of the overall look of objects (as measured by increase in the ratio of area of overlap to nonoverlap of normalized shapes of the objects) was obtained for basic level over superordinate categories. A significant but significantly smaller increase in similarity was obtained for subordinate over basic level categories.

\section{EXPERIMENT 4}

Experiment 4 explores one of the possible cognitive effects of the greater similarity in shape within basic level objects shown in Experiment 3. The hypothesis of Experiment 4 is that the basic level is the most general level at which an averaged shape of an object is identifiable as that object.

Experimental confirmation of the hypothesis requires two results. It is necessary to show that averages of different basic level objects which belong to the same superordinate category are not identifiable as members of the superordinate category - that is, that there is not a shape of an average furniture which can be identified as such. In addition, an average based upon the same sort of basic level object must be readily identifiable as such; that is, the average of shapes of two items from the same basic level category should be identifiable as to basic level or superordinate category membership.

\section{Methods}

\section{Subjects}

Subjects were 40 students in undergraduate psychology courses who volunteered to participate in the study for course credit.

\section{Stimuli}

To construct the averages, the two outlines to be averaged were overlapped in the standard orientation as described in Experiment 3. For all points in which the lines 
of the two outlines did not coincide, the point central between the two lines was taken and the points connected into continuous line segments.

These average drawings were constructed out of the same pictures paired, according to the same rules, as were used for computing the ratios of overlap in Experiment 3. One picture from each basic level category was randomly eliminated. This yielded 32 average drawings consisting of averages of objects within the same basic level category and 32 average drawings consisting of averages of two objects from different basic level but the same superordinate categories. Within the set of within-basic-level averages, six items were averages of objects within the same subordinate category and six were averages of one of those subordinates combined with the other object from the other subordinate class within the basic level category. Tracings of the averaged shapes were copied and given individually to the subjects.

\section{Procedures}

Twenty subjects received the 32 averages of the superordinate categories and 20 different subjects the 32 averages of the basic level (which included the subordinate) categories each in a different random order. Instructions for the basic level set were to "circle the category to which you think the object belongs" and to "write in your best guess about what the object is after the name of the category which you have circled." (The text of the instructions is a vailable in Roschet al., Note 1.) The four possible categories plus four others to which no objects belonged were listed on each page under the drawing. For superordinates, these were: animal, building(s), clothing, furniture, part of a human body, plant, tool, and vehicle.

Instructions for the superordinate set were the same, with the exception that subjects were told that the pictures consisted of averages of two different objects and that after they had circled the category to which they believed the object belonged, they were to write in guessed names of the two objects of which the outline they were seeing might have been constructed.

\section{Results and Discussion}

The mean number of correct identifications of superordinate categories per subject was 11 , i.e., approximately one-third. Since only one-eighth would have been expected by chance, subjects could clearly identify the category from superordinate averages better than chance. However, our pictures consisted of averages of only two outlines, and some of the individual objects remained identifiable at the basic level even when combined with objects of a different basic level category. If superordinate categories are to be shown identifiable as such, identification should be superior to chance even in those cases when neither of the basic level objects which composed the average was identifiable from the average drawing. To test this, each subject's actual number of correct superordinate category choices made when a basic level object was not named correctly was compared with the number of choices he could have made correctly by chance (one-eighth of the number of items for which that , subject did not correctly name a basic level object). A Wilcoxon MatchedPairs Signed-Ranks test of the difference between these two figures for the 20 subjects was not significant. Thus, it can be concluded that 
subjects' identifications of the superordinate category membership of averaged shapes was not superior to chance when the shapes composing the average were of different basic level objects and when neither basic level object was itself identifiable. From this experiment there is no evidence for visual features which make superordinate categories identifiable as such.

The mean number identifications correct for the basic level averages was 27 for superordinate category identification (chance would be 4 ) and 25 for basic level name $(78 \%)$. For all 32 stimuli, for both superordinate categories correct and basic level name correct, every basic level average shape was guessed correctly more times than its matched superordinate average shape, a result highly significant by any means of analysis.

The same subjects made both basic level and subordinate classifications. The mean correct items per subject were: for superordinate category identification-within subordinate 5.1, across subordinate 5.2; for basic level name identification - within subordinate 5.1, across subordinate 4.9. For neither type of identification was the Wilcoxon Matched-Pairs Signed-Ranks test significant. Thus, although in Experiment 3, within subordinate shapes obtained greater ratios of overlap than cross-subordinates shapes, subjects gave no evidence that they could identify subordinate level averaged shapes better than basic level averaged shapes.

In summary, averages of superordinate objects could not be identified as such better than chance; basic level objects were the most inclusive categories at which objects were readily identified. Furthermore, subordinate object averages were no more identifiable than were the basic level averages.

\section{CONCLUSIONS TO PART I}

The four experiments of Part I explore the interrelated aspects of the correlational structure of objects: clusters of co-occurring attributes common to the class, sequences of motor movements common to typical use or interaction with the object, physical similarity in the shape of the object, and high identifiability of averages of shapes of objects of the class. These four aspects of basic objects provide converging operational definitions of basic objects. For all of the taxonomies studied, regardless of whether language dependent variables such as attributes or language independent variables such as shape were used, there was a level of abstraction at which all factors co-occurred and below which further subdivisions added little information.

The focus of Part I was, in one sense, ecological. Psychological processes were only of indirect concern in these experiments. Basic objects, however, have a number of direct implications for psychological processes. Some of these psychological implications are explored in Part II. 
After these implications are explored, we return to a discussion of some of the unresolved issues arising from the converging operational definitions of basic objects of Part I-questions of the extent to which basic objects mirror real world structure and the extent to which ignorance and expertise can alter what constitutes a basic level category.

\section{PART II: SOME IMPLICATIONS OF BASIC LEVEL OBJECTS}

\section{Outline of Experiments 5 through 12}

Experiments 5 and 6 -cognitive representations. In these experiments, we tested the hypothesis that basic object categories are the most inclusive categories which can be represented by a code concrete enough to be called an image. The demonstration that basic objects are the most inclusive categories for which it was possible to recognize an averaged shape of members in Experiment 4 raises the possibility that basic objects are also the most inclusive taxonomic level at which it is possible to have a representation which is isomorphic to the physical appearance of objects of the class - in short, to have an image of the class. Experiment 5 seeks more direct proof of the nature of categorical representation. We did this by testing for perceptibility of visually presented objects after an auditory cue of the name of the object. The name of the basic level should be the most general level of abstraction for which prior presentation of the name of the category could aid perception of an object. Experiment 6 tests the same hypothesis using the priming paradigm in a same-different matching task (Beller, 1971; Rosch, 1975a, 1975b).

Experiment 7-object recognition. To test the hypothesis that objects are generally first seen or recognized as members of their basic level category, subjects were asked to indicate whether pictures of objects were correctly or falsely designated by names presented immediately prior to the pictures. If objects are first recognized as members of their basic level categories, such decisions should be made more rapidly to basic object names than to superordinate or subordinate category names.

Experiments 8 and 9-the development of categories. If basic objects are the categories most definable by means of visual perception and by sensory motor interaction with the object, they should be the first divisions of the world learned as categories by the young child. One developmental implication is that basic objects should be the first categories to be sorted in an adult taxonomic fashion by children and should be sorted taxonomically long before the superordinate combinations generally used in such tasks. This hypothesis was tested in Experiments 8 and 9.

Experiments 10, 11, and 12-language and categorization. Basic ob- 
jects should be the level of abstraction at which it is generally most useful to refer to objects. Thus, in Experiment 10 we tested whether basic object names are the lexical items normally chosen to refer to any given concrete object, and in Experiment 11 whether names for basic objects are the first concrete nouns acquired by children. If basic categories are the level of abstraction at which it is generally most useful to refer to objects, one would expect, in the evolution of languages, that names would evolve first for basic level objects, spreading both upwards and downwards as taxonomies increased in depth. A limited version of this hypothesis is tested in Experiment 12 for speakers of the American Sign language (of the deaf).

\section{EXPERIMENT 5: SIGNAL DETECTION}

Previous work on the effects of imagery on perception has shown that imagery can improve detection of similar signals (Peterson \& Graham, 1974), although imagery reduces detectability under conditions in which the signals are quite different from the image (Segal, 1971; Segal \& Fusella, 1970). Peterson and Graham found that subjects were more accurate in detecting pictures embedded in visual noise when they were shown the pictures and cued with the name of the picture prior to presentation than when they were not given advance information. Since Peterson and Graham's subjects saw the unmasked pictures, their study provides no information about the nature of the representation which subjects might generate solely from hearing the name of an object.

In this experiment, subjects were given the name of the object prior to each trial. If basic objects are the most inclusive class at which it is possible to have a representation which is isomorphic to the physical appearance of objects of the class, hearing the basic level name of an object may aid in the detection of a picture of an object of that category even when subjects have not previously seen that particular picture. Superordinate names should not aid in detection and subordinate names should not be more effective than basic level names.

\section{Method}

\section{Subjects}

Subjects were 80 students in psychology classes.

\section{Stimuli}

Pictures of all the objects in the nine taxonomies of Table 1 were obtained and pilot tested to ensure that there was complete agreement by subjects for their subordinate, basic, and superordinate names. Because there was ambiguity concerning the level of basic objects in the three biological taxonomies and because subjects from our student population 
were generally unable to identify items from the biological taxonomies below the level of tree or fish (see Experiment 10), only the nonbiological taxonomies were used in this experiment.

A total of 18 pictures were used, one for each of the three objects in the six nonbiological superordinate taxonomies. The subordinate level names for these pictures, arranged by the six groups of superordinate taxonomies are: livingroom chair, floor lamp, kitchen table; four-door sedan, city bus, tractor-trailer truck; double knit pants, knee socks, dress shirt; claw hammer, cross-cutting handsaw, regular screwdriver; delicious apple, cling peach, green seedless grapes; folk guitar, grand piano, and bass drum.

The pictures were $6.35 \times 8.89 \mathrm{~cm}$ color photographs mounted on $12.70 \times 20.32 \mathrm{~cm}$ white cards. Each picture was mounted randomly on the right or left side of the card with an equal number of pictures on each side. There was a $6.35 \times 8.89$ abstract drawing (in color) mounted on the side opposite the picture on each card. These provided some approximation to the colors and patterned areas of the target picture with which they were paired.

\section{Procedure}

Each card was displayed for $200 \mathrm{msec}$ in a two-field Harvard type tachistoscope. The cards were viewed through a mask which was directly in front of the card. The mask was constructed of random shapes cut from colored transparencies which were overlapped randomly. Where several transparencies overlapped, the area became completely opaque; areas of double and single transparency blocked perception of the stimulus to varying degrees. The effect was to interrupt perception of the stimulus pattern markedly both by interferring shapes and colors. Between trials, subjects were instructed to fixate a cross which appeared in the center of a $12.70 \times 20.32 \mathrm{~cm}$ white field. The exposure field and the pre- and post-exposure fields were illuminated by two 6-W fluorescent tubes concealed from the subject. Viewing distance was $33 \mathrm{~cm}$.

The subject's task was to determine on which side of the card the picture of the object appeared. They were told in advance that the target picture would appear equally often on the right and left. Subjects pressed a telegraph key with their right hand if they thought the object was on the right, and a telegraph key with their left hand if they thought it on the left.

There were four groups of 20 subjects. Subjects in the first group were told only that a picture of a common object would appear on one side or the other; the second group heard an auditory presentation of the superordinate name prior to each trial; the third group heard the basic level name prior to each trial; the fourth group heard the subordinate name prior to each trial. The category name (or a warning signal for the control group) was presented $1 \mathrm{sec}$ prior to stimulus onset. Subjects were told in advance that each picture would be a different object and that no picture would be repeated.

The mask and viewing interval had been adjusted hy pretesting so that a hit rate of approximately $70 \%$ would be achieved in the base condition in which no advance information was supplied. Subjects were given one practice card and one run through the 18 experimental cards. Subjects were tested individually; testing time was less than $10 \mathrm{~min}$ per subject.

\section{Results}

Mean percentage correct for the four conditions were: No information, 81; superordinate name, 69 ; basic level name, 90 ; subordinate name, 88 . The significance of these differences was tested by a two-day ANOVA (Condition $\times$ Category). Since category as well as subjects was a random 
variable, ${ }^{2}$ a Pseudo $\mathrm{F}$ (computed by the conservative minimum method, Clark, 1973) was used. The effect of condition was significant: $\min F^{\prime}$ $(3,102)=5.36, p<.01$. A Tukey test of differences between the means showed that both the basic level and subordinate name conditions were significantly different from the no-cue and superordinate name conditions but that the former two and latter two conditions did not differ significantly from each other. The hypothesis of the experiment was confirmed: Basic level categories aid in detecting a picture; superordinate names do not, and subordinate names aid detection no more than basic level names.

\section{EXPERIMENT 6: PRIMING}

The technique of priming in a matching task has been used to study the nature of mental representations generated by names for letters (Beller, 1971), colors (Rosch, 1975b), and superordinate semantic categories (Rosch, 1975a). Priming refers to presentation of a cue prior to the stimulus: Experiment 5 is an example of priming object identification by names. In a matching task, subjects are asked to determine whether two stimuli are the same or different. Same can be defined to mean physical identity or category identity.

Rosch (1975a) found that priming selectively influenced responses to pairs of items which were members of superordinate semantic categories when same meant the same category but that priming had no effect at all when same meant physical identity. With color names, however, she found that priming affected responses both under same-category and physicalidentity instructions. It would appear that subjects can generate a representation (or image) sufficiently isomorphic to the physical stimulus to aid responses under physical identity instructions only from a color name, not from a superordinate category name. Superordinate categories appear to include such visually diverse items that there can be no representation isomorphic to the physical features of the category as a whole. (In fact, subjects who claim to be able to image a superordinate category like furniture, invariably report doing so by imaging a particular item such as a chair or by imaging a symbol such as three blue triangles). In terms of the present account, basic level names should aid matching responses under physical identity instructions.

Two subexperiments were performed. In one, the conditions of Rosch (1975a) were replicated with the change that the basic names of items,

${ }^{2}$ In Experiments 5 through 7, Category was treated as a random variable in order to provide a conservative statistical test of the results. The categories used in the experiments were chosen to meet specific criteria and, thus, not entirely random. Furthermore, results of these experiments are intended to generalize only to concrete, visual objects not, for example, to auditory experience or abstract concepts. 
rather than the superordinate category names, were used for priming. In the second, the pictures used in Experiment 5 (which can be named at all three levels of abstraction) were used as stimuli; separate groups of subjects were primed with the superordinate, the basic level, and the subordinate category name.

\section{Methods}

\section{Subjects}

Sixty-five subjects were tested; all were students in psychology classes who received course credit for their participation. Twenty were tested with line drawings, 45 with pictures.

\section{Stimuli}

Line drawings. These drawings were the same used by Rosch (1975a). They were simple line drawings taken from elementary reading instruction materials. They tended to be such general representations of the basic category that they could not be further categorized at the subordinate level (e.g., an apple could not be identified as any particular type of apple). Each picture was of one typical or one atypical member of each of five superordinate categories. These were: furniture (chair, rug), vehicle (car, sled), fruit (apple, watermelon), vegetable (carrots, onions), tool (saw, hatchet). Pictures were mounted in pairs, centered, on $12.70 \times 20.32 \mathrm{~cm}$ white cards. The two pictures were spaced $0.635 \mathrm{~cm}$ apart. Viewing distance was $33 \mathrm{~cm}$.

Each picture occurred once in a physically identical pair (the same picture repeated) and once paired with a different picture. Thus, there were 10 same pairs and 10 different pairs, a total of 20 pairs of stimuli. Each occurred once primed and once unprimed for $\mathbf{4 0}$ presentations.

Color photographs. The pictures were the three items from the six superordinate categories used in Experiment 5. The pictures were mounted in pairs on $12.70 \times 20.32 \mathrm{~cm}$ white cards. Viewing distance was $33 \mathrm{~cm}$. Each pictured object was presented once in a same pair and once paired with a different picture. The same pairs were identical prints of the same photograph. There were, thus, 18 same pairs and 18 different pairs, a total of 36 pairs of stimuli. Each occurred once primed and once unprimed.

\section{Procedure}

For the line drawings, 10 subjects were primed with the superordinate category name, 10 with the basic name. For the color photographs, 15 subjects were primed with the superordinate name, 15 with the basic name, and 15 with the subordinate name.

The apparatus was as described in Experiment 5. Each stimulus pair was presented twice, once primed by the superordinate semantic category name spoken by the experimenter in advance of the pair, once preceded by the word blank (to equate for the prime's function as a warning signal). An interval of $2 \mathrm{sec}$ occurred between the spoken word and presentation of the visual stimulus which remained in view until terminated by the subject's response. Primed and unprimed trials alternated. During the period preceding presentation of the stimulus, the subject was asked to fixate a cross which occurred in the center of a white $12.70 \times 20.32 \mathrm{~cm}$ white field. The exposure and pre-exposure fields were illuminated by two 6-W fluorescent tubes; the post-exposure field was dark.

Subjects responded by pressing one of two response keys, one for each hand. Subjects were told to press the same key only when the members of a pair were physically 
identical and to press the different key to all other pairs. Subjects used their dominant hand for the same key. They were told to respond as rapidly as possible without error. It was emphasized to subjects that they would not be "fooled" by small difference between stimuli, that different pairs would be obviously different objects and that, if a pair appeared identical at first glance, it was identical. Reaction times were measured from the onset of the stimulus to the response.

A practice session preceded the beginning of testing. Practice items were drawn from the same categories but were not items used in the test sets. Practice sets contained 10 stimuli for the line drawings, 18 for the color pictures. The practice session familiarized subjects with the nature of the stimuli, the judgment required of them, and the mechanics of the apparatus and of making the response. Subjects received feedback on correctness and response time during practice.

In test trials, each subject received in different random order all pairs, primed and unprimed, from all categories: a total of 20 items for the line drawings, 36 for the color pictures. A test session lasted $1 / 2$ to $1 \mathrm{hr}$.

\section{Results}

The line drawings were analyzed by three-way ANOVAs for the variables Primed-unprimed (a within-subject fixed effect) $\times$ Good-bad example of the superordinate category (a within-subject fixed effect) $\times$ Category (a random variable). Because category as well as subjects was treated as a random variable, pseudo $F$ s (Clarke, 1973) were computed.

Reaction times for the same responses are shown in Table 6. There were no significant effects of priming with the superordinate name. A significant effect of priming for same responses primed with the basic level name was obtained: $F^{\prime}(1,6)=6.20, p<.05$. Whether the item was a typical or atypical example of its superordinate category did not reach significance. None of the effects for the different stimuli were significant. Error rates were low (a mean of $2.7 \%$ ) and did not differ significantly for any of the conditions. Thus, the basic hypothesis for the line drawing

\section{TARIF 6}

Reaction Times (Msec) for Same Responses in Priming Studies (Experiment 6)

Type of prime

Condition $\quad$ Superordinate name level name $\quad$ Subordinate name

Line drawings

$\begin{array}{lll}\text { Primed } & 629 & 579 \\ \text { Unprimed } & 615 & 633\end{array}$

Color photographs 
stimuli was confirmed; responses of same, when same was defined to mean physical identity, were unaffected by priming with the superordinate category name but were significantly facilitated by advance presentation of the basic level name.

The design for analysis of the groups who received the color photographs was a two-way ANOVA (Primed-unprimed $\times$ Category) performed separately for the same and different responses and separately for the groups primed with each level of category name. As in the case of the line drawings, pseudo $F$ s were computed. Reaction times for the same responses are shown in Table 6. There were no significant priming effects for the group primed with the superordinate category name. For the group primed with the basic level name, the effect of priming was significant $-F^{\prime}(1,9)=6.83, p<.05-$ as it was for the subordinate name $-F^{\prime}(1,8)=5.59, p<.05$. In order to test whether the effects of priming with the basic level and subordinate name differed significantly, a three-way ANOVA was performed in which the variable Basic namesubordinate name (a between-subject fixed effect) was combined with the variables already tested. The effect for basic versus subordinate name was not significant. None of the effects for different responses reached significance.

\section{SUMMARY OF EXPERIMENTS 5 AND 6}

The purpose of Experiments 5 and 6 was to bring together two lines of research: analysis of the properties of categories at different levels of abstraction and analysis of the nature of the cognitive representation generated by the category name.

Experiment 5 showed the basic level name of an object to be the most abstract name which could aid detection of a picture of the object in noise; Experiment 6 showed basic level names to be the most abstract category names which affected same responses under physical identity instructions. In both experimental paradigms, subordinate names were no more effective than the basic level names to which they were subordinate.

\section{EXPERIMENT 7: OBJECT RECOGNITION}

This experiment tests whether basic objects are first seen or recognized as members of their basic category (with additional processing required to identify them as members of their superordinate or subordinate category). Subjects should be able to verify category membership in basic categories more rapidly than membership in superordinate or subordinate categories. Subjects were presented with the photographs of objects used in the previous two experiments and were asked to verify whether or not each was a picture of an $X$, where $X$ was either a basic level, superordinate, or subordinate name. 
This is the first experiment in this paper in which subordinate classifications should elicit poorer performance than basic level classification. Thus, this experiment is the first demonstration that basic objects have greater cognitive primacy than subordinate as well as superordinate classifications.

\section{Method}

\section{Subjects}

Subjects were 45 students in an introductory psychology class.

\section{Stimuli and procedure}

Stimuli were the same color photographs used in the two previous experiments. Pictures were mounted in the center of $12.70 \times 20.32 \mathrm{~cm}$ white cards. The apparatus and conditions of illumination were the same as described in Experiment 5.

Subjects were told that they would hear the name of a kind of object and immediately thereafter would see a picture. The subject was instructed to press a response key with the forefinger of his dominant hand if the picture was an object of the type named, and to press another key with his nondominant hand if the picture was not an object of that type. The object name was spoken $500 \mathrm{msec}$ before the picture was displayed. The stimulus remained in vicw until the subject had made his response. Reaction times were measured from the onset of the stimulus to the response.

Each of the 18 pictures was presented twice per run, once preceded by a correct name and once preceded by a false name. Pictures and true or false names were presented in a different random order to each subject. Subjects were instructed to respond as rapidly as possible without errors. The complete set of names and pictures were presented for three consecutive runs to each subject. The first two runs were considered practice; reaction time was measured from the third run.

One group of 15 subjects heard the superordinate name and verified superordinate category membership; a second group of 15 subjects verified basic level category membership; and a third group of 15 subjects verified subordinate category membership. All of the false names were taken from different superordinate categories than that to which the object belonged. Thus, for the picture of a chair, the false superordinate name might be fruit, the false basic level name apple, and the false subordinate delicious apple.

\section{Results and Discussion}

Table 7 shows the mean reaction time for verification of category membership for the three types of category name. A two-way ANOVAType of category name (a between-subject fixed effect) $\times$ Category (a random variable) was performed on the reaction time data. Category was treated as a random variable and data were analyzed by means of pseudo Fs (Clark, 1973). True pairs and false pairs were analyzed separately.

The results of the analysis confirmed that there was a significant difference between response times for the three different types of category name. For true pairs: $F^{\prime}(2,8)=9.71, p<.01$. A Tukey test showed that basic level category names were faster than either superordinate or subordinate names and that superordinate category names were faster than 
TABLE 7

Mean Reaction Times(Msec) for Verification of Category Membership of Objects

Type of name

\begin{tabular}{cccc}
\cline { 2 - 4 } Response type & Superordinate & Basic level & Subordinate \\
\hline True & 591 & 535 & 659 \\
False & 630 & 578 & 642 \\
\hline
\end{tabular}

subordinate. For false pairs: $F^{\prime}(2,6)=6.35, p<.05$. A Tukey test showed that basic level names were faster than either superordinate or subordinate names; however, for the false pairs, superordinates and subordinates did not differ significantly from each other. Error rate was low (2\%) and did not differ significantly by condition.

The basic hypothesis of the experiment was that objects could be identified more rapidly as members of their basic level category than as members either of their superordinate or subordinate category. The results clearly support that hypothesis. The nature of the additional processing required for superordinate and subordinate identifications cannot be established from the present experiment. We may speculate that after identification of the basic class of an object, superordinates are derived by inference from the class membership of the basic object and that subordinates are derived from observation of attributes - additional to those needed to perceive the basic object - which are relevant to subordinate distinctions. That processing is not identical for superordinate and subordinate identifications is indicated by the significant difference between responses to those types of name for the true pairs.

\section{EXPERIMENTS 8 AND 9: DEVELOPMENT OF CATEGORIES}

There is a long tradition of research which indicates that young children and adults classify objects in different manners. When given instructions to "put together the things that go together," adults tend to put things together taxonomically (e.g., objects that belong to the same category), whereas young children are likely to sort on the basis of complexive groupings-associations, stories, chains, and other nontaxonomic criteria (see, for example, Annett, 1959; Bruner, Olver, \& Greenfield, 1966; Denny, 1974; Garrettson, 1971; Goldman \& Levine, 1963; Thompson, 1941; Vygotsky, 1962). The stimuli used in sorting tasks have tended to be of two types: If abstract (e.g., geometric forms varying in dimensions such as form, color, and size), they are typically presented in a set which has no structure (e.g., each attribute occurs with all combinations of all others); if representational (e.g., toy versions or pictures of real-world 
objects), the arrays are typically such that they can be grouped taxonomically only at the superordinate level. Thus, the representational stimuli used in sorting tasks are such that if the child were to sort the objects into those of like taxonomic category, he would have to put together such items as socks and shirt, or dog and cow. Children do not seem to have been asked to sort together objects belonging to the same basic level category (e.g., several shoes or several dogs). We suspect this results from the fact that basic objects are so obviously the "same object" to adults that a task does not seem to be a problem of categorization to an adult experimenter unless objects are taken from different basic level categories.

The development of classification skills is a complex topic. The nature of the task appears to make a great difference. Even children as young as $2 \mathrm{yr}$ appear to possess some working knowledge of superordinate categories, if recall of categorically related versus unrelated items is the dependent variable (Goldberg, Myers, \& Perlmutter, 1974). And there can be no doubt that some aspects of the logic of categorization develop with age (for example, Bruner et al., 1966; Inhelder \& Piaget, 1956; Vygotsky, 1962). According to the present theory of categorization, the nature of the stimulus array should make a great difference. In terms of cue validity, basic objects are those that are the most differentiable and, thus, the generally most useful distinctions to make in the world. With respect to sensory-motor development, basic objects should be the first categories learned by means both of visual perception and sensory motor interaction with the object. With respect to imageability, basic objects are the most inclusive categories for which an image can be formed; if children encode the world by images or other iconic representations before they encode more symbolically (as argued, for example, by Bruner et al., 1966), basic objects should be learned easily by children on this ground also. In short, there are many reasons to suspect that development of basic object categories occurs early.

The purpose of the following two experiments is to replicate the design of previous sorting studies in which only superordinate classifications had been possible, but using stimulus materials which enable subjects to make basic level classifications. We believe that sorting into basic level categories is not dependent upon the development of mature adult naming or reasoning. We expected that basic level sorting would occur at the earliest ages and would be independent of superordinate sorting or of a child's ability to explain the categories.

In the following experiments, subjects at each of the following ages $-3 \mathrm{yr}, 4 \mathrm{yr}$, kindergarten, grades 1,3 , and 5 (ages 5, 6, 8, and 10), and adult - were divided into two groups. One group was given an opportunity to sort sets of color pictures of common objects such as animals, vehicles, clothing, and furniture into groups of basic level objects. 
The other group was given the same pictures, but in sets cross-cutting the basic level so that taxonomic sorting would necessarily be at the usual superordinate level. 'The 3- and 4-yr-olds received only two categories, and the task was administered in the form of oddity problems: the older groups received four categories, and the standard sorting task and instructions were used.

\section{EXPERIMENT 8}

While children below the age of 6 yr may have difficulty understanding instructions in the standard sorting task (Bruner et al., 1966; Nash, Note 2), oddity problems can be comprehended at much younger ages (Gelman, 1972). Thus, in order to be able to include nursery school age children in the experimental design, the first experiment employed an oddity problem format.

\section{Method}

\section{Subjects}

Subjects were 40 nursery school children, 40 elementary school children, and 20 adults. The children included 20 3-yr-olds, 20 4-yr-olds, 10 kindergarteners, 10 first graders, 10 third graders, and 10 fifth graders. In each age group, exactly half of the children were males and half females. Mean ages at the time of testing, for each age group, respectively, were: 3 yr, 5 months; 4 yr, 7 months; 5 yr, 7 months; 6 yr, 5 months; 8 yr, 8 months; and $10 \mathrm{yr}, 7$ months. The adult subjects (six males, 14 females) were undergraduates who participated in the experiment for course credit.

\section{Stimuli}

Stimulus materials were color photographs of animals and vehicles. Four categories of animals - cats, dogs, butterflies, and fish-and four categories of vehicles-cars, trains, motorcycles, and airplanes - were used. Pictures were selected from the pool of 100 or more pictures of objects from which the pictures for Experiment 3 were selected. Four pictures were used for each basic level category. Two sets of triads were used: one in which two pictures in each triad could be correctly paired at the basic level, and one in which pictures could be correctly paired only at the superordinate level.

To prepare the basic-level triad set, four pairs of pictures were composed from each basic level category yielding 16 pairs per superordinate for the four basic level categories. Pictures were paired, with the restrictions that each of the four available pictures per basiclevel category be used twice and that the two pictures in a pair not be the same color. The third member of the triad was chosen from the other superordinate category by the following procedure: given that there were four pairs of pictures within each basic level category, each of the pairs from the same basic level category was combined with one picture from each of the four different basic level pairs of the other superordinate. Thus, from the four pairs of cats, one pair was combined with a car, one with a truck, one with a motorcycle, and one with an airplane. The four pairs of fish were combined with a different car, truck, motorcycle, and airplane. This procedure was repeated for all eight categories, yielding 32 different triads.

To prepare the superordinate triad set, each picture from a basic level category was paired with one picture from each of the other basic level categories within the same 
superordinate. Thus, each car was paired with one train, one motorcycle, and one airplane. Again, the restriction was used that the two pictures in a pair not be the same color. This procedure yielded six sets of four pairs for each superordinate. Each pair was combined with one member from the other superordinate category according to the procedure described for basic level pairs. There were 48 triads in the superordinate set. For both the basic level and superordinate sets, all pictures were used an equal number of times.

\section{Procedure}

For the two nursery school groups, triads were presented to the child, one at a time. The three pictures were put on the floor, and the child was told to put together (point to) "the two that are alike, that are the same kind of thing." After the child had gone through the entire set, the last six triads were shown to the child, one at a time. He was reminded which two pictures he had put together and was asked why they belonged together. If the child gave any reason other than a taxonomic one, he was asked if there were any other reason why the two pictures belonged together. Triads were presented in a different random order to each subject, and the order of pictures within triads was shuffled between subjects. Half the subjects in each age group (10 subjects) performed the task with the basic level set and half with the superordinate set. The nursery school children and the adults participated in the entire experiment as outlined above. Because a ceiling in correct sorting of both basic level and superordinate pairs had been reached by age four, and because a ceiling in giving taxonomic reasons for sorts for the basic level had also been reached by age four, the other subjects (elementary school children) were required to sort only six randomly chosen superordinate triads, for which they were asked to give the reason for their sort.

\section{Results and Discussion}

The results were clear. At all age levels, basic level sorts were virtually perfect; for the 3-yr-olds, the percentage correct for basic level sorts was 99 , and for $4 \mathrm{yr}$ and older, basic level sorts were perfect. Performance was considerably lower for the youngest age group, however, on sorts of the triads which could only be paired at the superordinate level: 3 -yr olds, $55 \%$ correct; 4 -yr olds, $96 \%$ correct.

There were no sex differences; data for both sexes were, therefore, combined in the analyses reported. A three-way analysis of variance (ANOVA) was performed on the pairing responses for the triads. Between-subject factors were Grade (3-yr olds, 4-yr olds, and adult) and Type of category (basic or superordinate). Taxonomy (animal or vehicle) was nested within Type of category. The dependent variable was percentage of correct responses. The effects of primary interest were that of Type of category and the interaction between Type and Grade. The prediction that basic level sorts would be correct significantly more often than superordinate sorts was confirmed, $F(1,54)=26.58, p<.01$. Because a ceiling in performance on these simple triads had been reached essentially by age four, a significant interaction could be expected between Type of category and Grade; that result was also obtained, $F(2,54)$ $=18.54, p<.01$. These two results show that there do exist objects 
which even small children will classify in the same manner as adults. Basic level sorts are equally easy for all age groups; it is only superordinate level sorts which improve with age.

Not unexpectedly, the main effect of Grade was also significant: $F(2,54)=20.9, p<.01$. The effect of taxonomy (animal or vehicle) was not significant $-F(1,54)=.49$, ns - and no interaction other than that between Type of category and Grade was significant. A Tukey test confirmed that 3-yr olds performed significantly worse than either 4-yr olds or adults, while there was no difference in performance between the latter two groups.

From the results of the sorting alone, it might be argued that the findings are simply a function of language development; that is, that children learn the names for basic level objects before those for superordinate categories, and that items are put together when the child knows they have the same name. Two pieces of evidence from the present study argue against such an interpretation.

Subjects' reasons for six of their sorts were obtained for all age levels. These reasons were classified into taxonomic reasons (giving the name of the two items placed together) and all other reasons such as giving attributes or autistic responses. To demonstrate the failure of naming to account for sorting results, results for percentage of correct names for superordinate sorts are shown in Fig. 2. Adults are omitted because they were perfect in both sorting and naming.

Two points are made clear by Fig. 2. For all ages, correct sortings were superior to correct namings. (This was true also for the basic level names at the youngest age; correct sorts for 3-yr olds were $99 \%$, correct names 65\%; however, a ceiling was reached for basic level names by age four.) Since a difference between sorting and naming could conceivably have been due partly to guessing (correct sorts could sometimes have

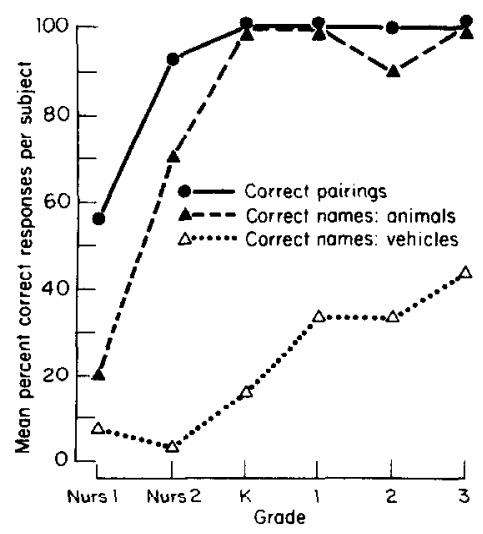

FIG. 2. Correct sorting and correct reasons in the triads task of Experiment 8. 
been produced by chance but correct names had to be generated from an infinite set of linguistic responses), correct names were compared for the animal and vehicle categories. As already reported, there were no differences in percent of correct sorts between the animals and vehicles; however, as Fig. 2 shows, animal pairs were named correctly far more often than vehicles at all age levels except the youngest, in which names for both were poor, and adults, in which names for both were perfect. The significance of this finding was tested by the Sign test (correct responses for animals versus vehicles for each child). Separate analyses were performed for each age level. The difference between animals and vehicles was not significant for the 3 -yr olds or adults but was significant for all other age levels: 4-yr olds, $p<.035$; kindergarten, $p<.004$; grade 1 , $p<.004$; grade $3, p<.016$, grade $5, p<.016$.

In summary: Pictures of objects classifiable into basic level categories were classified in an adult taxonomic manner by children at all ages, including 3-yr-old children. Only the sorting of superordinate level objects showed the usual improvement with age. Evidence was presented that these results are not simply due to difference in knowledge of names for basic and superordinate level categories.

\section{EXPERIMENT 9}

Experiment 8 used a simplified oddity problem format in order to creatc a meaningful task for 3- and 4-yr-old children. However, in most previous studies a different sorting procedure has been used. In order to use a task directly comparable to that of previous studies, and in order to have a task difficult enough to show sorting differences for children older than $4 \mathrm{yr}$, a second study was performed which was based on the same logic as Experiment 8, but which used a standard sorting format.

\section{Method}

\section{Subjects}

Subjects were 64 children and 16 adults. The children were 16 kindergarteners, 16 first graders, 16 third graders, and 16 fifth graders. In each group, exactly half the children were males and half females. Mean age at the time of test, for each group respectively was: $5 \mathrm{yr}, 7$ months; $6 \mathrm{yr}, 5$ months; $8 \mathrm{yr}, 4$ months; and $10 \mathrm{yr}, 7$ months. Adult subjects (six males, 10 females) were undergraduates who participated in the experiment for course credit.

\section{Stimuli}

Stimulus materials were color photographs of objects in four categories. Three of the categories were divided into superordinate and basic level classes: clothing - shoes, socks, shirts, pants; furniture - tables, chairs, beds, sofas; vehicles - cars, trains, motorcycles, airplanes. These pictures were from the same pool of pictures used in Experiment 3 and were chosen in the manner described in Experiment 8. In addition, another category, 
people's faces, was added because of its intrinsic interest and attention attracting qualities to children. This category was included in the sorting set in the same manner as the others, namely, people's faces - men, women, young girls, babies. There was reason to believe that these pictures belonged to a taxonomy which contained more than one possible basic level and that the tendency to sort peoples' faces together would be as strong as the tendency to sort men's faces, etc. together (Carey, DeVilliers, \& DeVilliers, Note 3). The human pictures were intended to provide an impetus to the children to sort both the superordinate and basic level sets taxonomically.

There were four sets of stimuli which could be sorted at the basic level and four sets which could only be sorted taxonomically into superordinate categories. Basic level sets consisted of one of the basic level categories from each of the four superordinates. For example, one subject might receive four shoes, four chairs, four men's faces, and four cars. Two subjects at each age level received each basic level set. To form the superordinate sets, each picture within the basic level sets was numbered arbitrarily 1, 2, 3, or 4. For a set, all of the like numbers were combined. For example, one subject received shoe 1 , sock 1 , shirt 1 , pants 1 , table 1 , chair 1 , car 1 , etc. Two subjects at each age level received each superordinate level set.

\section{Procedures}

The pictures in a set were shuffled and laid in front of the subject in random order. Instructions were: "Here are some pictures. (The experimenter called the child's attention to each picture by pointing to each in turn.) Put together the ones that go together, the ones that are the same kind of thing." The child was encouraged to include all of the pictures in his groupings. If his first sort was not taxonomic, the pictures were returned to a random order and he was asked if he could find another way to put them together. When the child had finished each sort to his satisfaction, he was asked why those pictures went together.

\section{Results and Discussion}

A child was considered to have sorted taxonomically if either his first or second sort was broken into four groups of four pictures corresponding to the four categories built into the stimuli. The pattern of results was very similar to that obtained in Experiment 8. As in Experiment 8 , there were no sex differences. For the basic level categories, all but one child in kindergarten and all but one child in the first grade sorted in an adult taxonomic fashion. For superordinate level sorts, however, only half of the children in each of those grades sorted taxonomically. All of the children in the older group (grades 3 and 5) and all of the adults sorted taxonomically for both basic level and superordinate sets. Because the results for kindergarten and first grade were identical, those two groups were combined for a $\bar{X}^{2}$ comparison of the difference between basic level and superordinate sorts. Results confirmed that for this group, basic level sets were sorted taxonomically significantly more than superordinate sets: $\bar{X}^{2}(1)=3.64, p<.05$. Thus, as had been the case for the oddity problems of Experiment 8, children of all ages were virtually perfect when sorting the basic level stimuli, and only showed the usual developmental trend in sorting the superordinate level categories. 


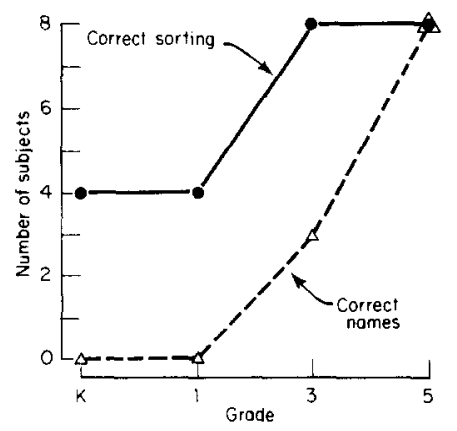

FIG. 3. Correct sorting and correct reasons in the sorting task of Experiment 9.

The children's reasons for their sorts were divided into taxonomic and nontaxonomic reasons following the procedures of Experiment 8 . The comparison of taxonomic sorts and reasons is shown in Fig. 3. The production of taxonomic reasons lagged behind taxonomic sorting responses. Separate $\bar{X}^{2}$ tests were performed for grades kindergarten and 1 and for grades 3 and 5 for the difference between the number of children giving taxonomic reasons versus the number giving taxonomic sorts. Both tests were significant: for the younger group, $\bar{X}^{2}(1)=18.66, p<.001$; for the older group, $\bar{X}^{2}(1)=3.79, p<.05$. In the present experiment, the probability of correct sorting by chance was extremely small (unlike the one-third correct guessing probability for the triads of Experiment 8). Thus, the difference between taxonomic sorts and taxonomic reasons indicates that the sorts were based on principles other than simple knowledge of the category names.

In summary: For a traditional sorting task, as well as for the oddity problems of Experiment 8, even kindergarten children sort in an adult taxonomic manner when given categories which can be sorted at the basic level. Developmental changes in sorting occur only for sets which can be grouped solely at the superordinate level.

\section{DISCUSSION OF EXPERIMENTS 8 AND 9}

Children can sort into basic level categories at the age of $3 \mathrm{yr}$ for an oddity problem procedure, and at the age of $5 \mathrm{yr}$ for a sorting procedure. Basic level sorting is independent of superordinate sorting and independent of the reasons given for the basic level sorts. Both superordinate sorting and reasons for sorts develop with age. Because basic level sorting was already at ceiling at the earliest ages studied, it was not possible to examine sorting of subordinate categories. It seems unlikely that sorting of subordinate classes will be performed earlier than sorting of the basic level classes to which they are subordinate; however, such a demonstration is left to future research. 
Because sorting into basic level categories is, by the nature of basic level objects, overdetermined, the present experiments do not distinguish among the possible bases on which children may have performed their sorts. In fact, in light of the present research, it would not appear to be a fruitful approach to ask whether children classify on the basis of form or function or attributes or category names or "similarity" or any other single criterion. (Nor does it appear fruitful to debate whether form or function is more primary in children's learning-e.g., Anglin, 1976; Nelson, 1973.) A consistent finding of the present research project has been that these factors are not independent. Children are probably engaged in learning the co-occurrence contingencies of their environment and probably categorize on the basis of their knowledge of those contingencies. Experiments 8 and 9 are a first step in demonstrating the primacy of basic level categories in development; further specification of the complex processes by which environmental structure (and adult categorizations) become internalized in children requires additional research.

\section{EXPERIMENTS 10, 11, AND 12: IMPLICATIONS FOR LANGUAGE}

It would have been possible to begin our study of basic objects by an examination of language. For example, Berlin (see Berlin et al., 1973) used linguistic criteria to define generic (basic) level categories in plant taxonomies.

The present theory of categorization has a number of implications for language. First, in most situations, the distinction for which a lexical item is needed should be a distinction encoded by a basic level name. Thus, object names at the basic level of abstraction should be the names by which objects are most generally designated by adult speakers of the language. Experiment 10 tests this hypothesis. Second, basic level names should be the first linguistic labels for objects acquired by the child. This hypothesis is tested in Experiment 11 by use of the developmental linguistic protocol's of Roger Brown's Sarah (Brown, 1974). Third, if a language lacks taxonomic depth in domains of concrete objects, it can be predicted that basic level classes will be present and that it will be superordinates and/or subordinates which are lacking. Because environment and culture partially determine the attributes on which basic categories are structured, such a hypothesis is difficult to test. It proved possible, however, to test the hypothesis in a limited manner by a study of some object taxonomies in American Sign Language of the Deaf, a language spoken (signed) by people sharing the same basic material culture with speakers of standard English. This test is performed in Experiment 12. 


\section{EXPERIMENT 10}

\section{Method}

\section{Subjects}

Subjects were 130 students in psychology classes.

\section{Stimuli and Procedures}

Stimuli wcre $6.35 \times 8.89 \mathrm{~cm}$ color pictures of all of the objects in the nine taxonomies of Table 1 . There were two parts to the experiment. In the first part, subjects named the pictures; in the second part, we verified that individuals from the same subject population did, in fact, know the superordinate and subordinate names.

Subjects were instructed first to leaf quickly through the entire packet of pictures, then to turn back to the first page and go through the packet again, this time writing under each picture, the name of the object. (Complete instructions are available in Rosch et al., Note 1).

Ten copies of each picture were used. The prints, in plastic envelopes, were inserted into the pages of a packet on which subjects wrote the names. Three types of packet were used: those in which one picture from each superordinate category appeared (a total of nine items per subject); one in which one picture from each basic level category appeared (a total of 27 items per subject); and one in which all pictures appeared (a total of 54 items per subject). These represented three different contrast sets: one in which the superordinate name was sufficient to identify each item from among the others, one in which the supposed basic level name was needed to identify each item, and one in which only the subordinate name would distinguish each item from the others.

A total of 10 subjects named each item; this meant that a total of 60 subjects named items in the superordinate contrast sets, 20 subjects in the basic level contrast sets, and 10 subjects in the subordinate contrast set.

Because a tendency to name at the basic level might be attributed to lack of knowledge of superordinate and subordinate terms, the second part of the study investigated subjects' knowledge of these names. For the superordinate names, subjects received all of the 54 items with the statement under each, "This is an X" where $\mathrm{X}$ was the correct superordinate name for half of the pictures and an incorrect superordinate name for half of the pictures. Subjects checked "True," "False," or "Don't know." Ten subjects received each picture with a true, and 10 with a false superordinate name; thus, 20 subjects participated in this condition. Due to the results for the biological categories in the previous experiments (and the results of the naming portion of this experiment), the superordinate names used for the biological categories were plant and animal.

Verification of subordinate names was performed by mounting pictures of the two subordinates of a basic level category side by side. The correct subordinate name of one of the pictures was printed underneath, and subjects were asked to check the picture correctly described by that name. The correct name appeared 10 times for each item of the pair. A total of 20 subjects participated. For this condition, the names for items in the biological categories were at the level shown as the basic level in Table 1.

\section{Results and Discussion}

Results for the naming are shown in Table 8. Regardless of contrast sets, subjects overwhelmingly used the basic level name in this free-naming situation. 
TABLE 8

Type of Name Given in Free Naming of Pictures

Type of name given

\begin{tabular}{lcccc}
\cline { 2 - 5 } Contrast set & Superordinate & Basic level & Subordinate & Other \\
\hline Superordinate & 0 & 532 & 5 & 2 \\
Basic level & 0 & 533 & 4 & 2 \\
Subordinate & 1 & 530 & 5 & 4 \\
\hline
\end{tabular}

There are two possible claims that these results might be artifacts of linguistic facts other than level of classification. One claim is related to word frequency. It may be suggested that what are here called basic level names are simply more frequent than superordinate or subordinate names, and it can thus be argued that, in this name production task, subjects simply replied with the most available (frequent) word for that object. While word frequencies are not obtainable for the subordinate classes (because they are generally phrases, not single words), word frequencies were available for superordinate and basic level names in five taxonomies. In nine of the 15 cases of superordinate-basic level comparison, the superordinate name actually had a higher word frequency than the basic level name; however, in these cases subjects showed no greater tendency to name with the superordinate than in the cases where the basic level name was more frequent -in both situations, as shown in Table 8, the tendency to name with the superordinate was virtually nonexistent.

The naming results might also be attributed to the subjects' linguistic ignorance. Subjects simply might not know the correct superordinate or subordinate name. The second part of the study tested this possibility. For superordinate names, there were no errors at all in any of the truefalse judgments. For the subordinate level identifications, for the nonbiological categories, 16 of the 18 pairs (32 subordinates out of 36 ) received errorless identification. For the biological categories, there was confusion with oak and maple trees and errors with all the fish. The three birds were distinguished with no errors. Thus, the overwhelming use of the basic level name can not be attributed to ignorance of correct designations for items at other levels of abstraction.

In summary: There was virtually total agreement in the use of basic level names for 54 objects from nine taxonomies. It was shown that these results were not an artifact of word frequency or lack of knowledge.

\section{EXPERIMENT 11}

This experiment examines the hypothesis that names at the basic level should be the first concrete nouns acquired in the language development of 
TABLE 9

Concrete Nouns Used in Stage I of Language Acquisition

\begin{tabular}{|c|c|c|c|c|c|c|}
\hline \multirow[b]{3}{*}{ Category } & \multicolumn{6}{|c|}{ Taxonomic level of word used } \\
\hline & \multicolumn{2}{|c|}{ Superordinate } & \multicolumn{2}{|c|}{ Basic level } & \multicolumn{2}{|c|}{ Subordinate } \\
\hline & Tokens" & Types" & Tokens & Types & Tokens & Types \\
\hline \multicolumn{7}{|c|}{ Nonbiological } \\
\hline $\begin{array}{l}\text { Musical } \\
\text { instrument }\end{array}$ & 0 & 0 & 13 & 6 & 0 & 0 \\
\hline Fruit & 0 & 0 & 7 & 3 & 0 & 0 \\
\hline Tool & 0 & 0 & 37 & 13 & 0 & 0 \\
\hline Clothing & 2 & 1 & 91 & 18 & 4 & 1 \\
\hline Furniture & 0 & 0 & 75 & 16 & 1 & 1 \\
\hline Vehicle & 0 & 0 & 50 & 11 & 3 & 2 \\
\hline \multicolumn{7}{|c|}{ Biological $^{b}$} \\
\hline Tree & 0 & 0 & 0 & 0 & 0 & 0 \\
\hline Fish & 19 & 1 & 0 & 0 & 0 & 0 \\
\hline Bird & 13 & 1 & 0 & 0 & 0 & 0 \\
\hline
\end{tabular}

"The token count is the total number of utterances of any word of that classification; the type count is the number of different words in the classification which were used.

${ }^{b}$ The level shown as superordinate matches Table 1 but is the level shown to be the basic level for biological taxonomies in the earlier experiments.

a child. One well documented case history was taken for analysis; the protocols for the spontaneous speech of Sarah (Brown, 1974). The corpus analyzed was 2 -hr weekly recordings of Sarah's spontaneous speech during her initial period of language acquisition (all of her utterances in Stage I; see Brown, 1974, for definitions of the stages in child language acquisition).

Two raters read Sarah's protocols. All utterances of an item in any of the nine superordinate taxonomies previously studied were recorded. Repetitions of an adult's utterance or of Sarah's own utterance were not included. These utterances were classified as superordinates (the superordinate term itself or any synonym); basic level (any word on the same level of linguistic contrast as the basic level items in Table 1-see Frake, 1969, or Rosch \& Mervis, 1975, for definitions of linguistic contrast); or as subordinates (any term on the same level of linguistic contrast as the subordinate terms in Table 1).

The results of the study are shown in Table 9. Results were sufficiently extreme as to render statistical analysis unnecessary. Both in total number of utterances of any word in a classification and in number of different words in the classification used, basic level names were essentially the only names used by Sarah in Stage I. 
This was not an artifact of simple word frequency. More than four times as many basic level terms which had lower frequencies than their superordinate occurred in Sarah's protocols than basic level names which had higher frequencies than the superordinate. (If adults use primarily basic level terms in talking to children, that supports our argument.)

Additional support for the primacy of basic level names in children's acquisition of concrete nouns comes from a study of the names given by children for the pictured objects used in Experiment 10. Thirty 3yr-old children were each asked to name nine of those pictures. Of the total of 270 names given, only one item was not a basic level name. Although not all of the names provided by the children were correct, errors were not words at higher or lower levels of abstraction than the basic; they were basic level names for objects other than those pictured-for example, the grapes were frequently called blueberries.

In summary: For one intensively recorded case study of language acquisition, it was found that essentially all of the child's first utterances of concrete nouns in the nine most frequent categories of such nouns in English were at the basic level of abstraction. Furthermore, young children, like the adult subjects in Experiment 10, used basic level names almost exclusively to name objects in pictures.

\section{EXPERIMENT 12}

This experiment examines an issue dealing with language function and evolution and is therefore quite speculative. The hypothesis is that basic categories are the most necessary in a language. If that is the case, when taxonomic depth is reduced in some domains in some languages, it should be basic level objects which will be coded and names for superordinate and/or subordinate categories which will be lacking.

Although deaf Americans live in the same environment of concrete objects possessing the same correlational structure as do hearing Americans, their sign language appears to have fewer fixed signs for concrete objects than does spoken English. This appears to be due to the capability in Sign for other forms of communicative elaboration (see, for example, Klima \& Bellugi, 1975). Thus, American Sign Language of the Deaf (ASL) appeared to be an ideal case on which to test our hypothesis. ${ }^{3}$

The study was performed by the fifth author, herself a fluent speaker of ASL. Four informants were used: three deaf individuals whose native language was ASL and one hearing linguist fluent in ASL. Informants were interviewed extensively concerning the existence of signs for the items at each of the levels of abstraction in the taxonomies in Table 1. An effort was made to determine those categories for which a consistent

\footnotetext{
${ }^{3}$ It should be noted that ASL is a true language of its own. It is not simply English translated into signs. English words can be finger-spelled, but this is not a part of ASL (Klima \& Bellugi, 1975).
} 
sign or sign combination was used (as in the spoken English), and those categories which did not appear to be coded at all in ASL (e.g., were communicated, if at all, by a lengthy explanation or by finger spelling of the English word). In computing the results, a consistent sign or sign combination was considered to exist if any one of the four informants thought it existed.

The results are shown in Table 10. Because there were different numbers of categories at the different levels of abstraction, percentages of the possible terms are shown. A one-way ANOVA was performed for the difference between the percentage of superordinate, basic level, and subordinate signs for the nonbiological taxonomies. The six nonbiological categories served as the random variable in the analysis. Results were: $F(2,5)=16.37, p<.01$. A Tukey test showed there were a significantly greater percentage of signs at the basic level than at either the superordinate or subordinate, and that there were significantly more signs at the superordinate than at the subordinate levels. Although there were too few biological categories to analyze statistically, it is clear that, as was the case for hearing subjects, the supposed superordinate appears to be the basic level for these taxonomies.

The present experiment confirmed that, in one language which lacks some of the terminology for concrete objects which exists in standard English basic level terms were almost as common as in English, whereas, superordinate and subordinate terms were significantly less common. Thus, this study demonstrates in a limited way, the primacy of lexical items for basic level categories in language.

In addition, the present study raises a more general issue. Why does standard English need designations for superordinate and subordinate categories for those domains in which ASL does not? Why are hierarchical classification systems found in language at all? A more detailed analysis of classification and use of language in the deaf community using ASL may provide clues for the more general question. In addition, examination of taxonomies in other languages (when possible, in historical

TABLE 10

Percentage of Items Having Consistent Signs or Sign Combinations in ASL

\begin{tabular}{lccc}
\hline & \multicolumn{3}{c}{ Taxonomic level } \\
\cline { 2 - 4 } Category type & Superordinate & Basic level & Subordinate \\
\hline Nonbiological & 33 & 90 & 8 \\
Biological" & 100 & 22 & 0
\end{tabular}

"The level shown as superordinate matches Table 1 but is the level shown to be the basic level for biological taxonomies in the earlier experiments. 
depth) might prove extremely enlightening; from study both of occurrences of taxonomic depth in languages and of the evolution and degeneration of taxonomic depth, we may gain a great deal of knowledge of the functions and development of languages.

The preceding three experiments demonstrate that the names of categories shown to be at the basic level of abstraction are the names which are most used by adults, which are first used by children, and which appear to be the names most necessary in a language. Of course the existence and use of those lexical items is itself an important factor in cognition, but that is a different issue.

\section{PART III. GENERAL DISCUSSION}

The categorizations that humans make of the concrete world are not arbitrary, but rather are highly determined. They are determined, in the first place, because the perceived world is not an unstructured total set of equiprobable co-occurring attributes. Unlike the artificial stimulus arrays typically used in concept identification research, the material objects of the world possess high correlational structure. Categories are determined, in the second place, because, in so far as categorization occurs to reduce the infinite differences between stimuli to behaviorally and cognitively useful proportions, the basic category cuts in the world should be those which yield the most information for the least cognitive load. Category cuts should provide the most inclusive categories which can follow the correlational structures perceived in the world. Basic objects are the categories at the level of abstraction for which the cue validity of categories is maximized. Categories at higher levels of abstraction have lower cue validity than the basic because they have fewer attributes in common; categories subordinate to the basic have lower cue validity than the basic because they share most attributes with contrasting subordinate categories.

The four experiments performed in Part I of the study explored some of the interrelated aspects which make up the correlational structure of objects: clusters of co-occurring attributes common to the category, sequences of motor movements common to typical use or interaction with the object, objective similarity in the shape of the object, and identifiability of an average shape of objects in the class. For all of the taxonomies studied, there was a level of abstraction at which all of these factors co-occurred and below which further subdivisions added little information. ${ }^{4}$

${ }^{4}$ It should be emphasized that these claims are made with regard to concrete not abstract objects. To the extent to which categories are not concrete, the overall look of an object and the motor programs for using it may not be necessary concommitants of the attributes and functions of the object. This is true of concrete objects used abstractly as well 
The eight experiments performed in Part II explored implications of the concept of basic objects. Two major implications were economy of representations and the general usefulness of categories. In experiments on visual detection and priming of classification, the basic level was shown to be the most abstract level at which perceptual identification of an object could be aided. An experiment on the verification of category membership of pictures of objects indicated that objects are first seen and recognized as members of their basic category. Children were shown to sort basic objects taxonomically even at ages where other stimuli have failed to elicit such classification. Basic object names were shown to be the most used in language by adults, the first used by children developing language, and the least dispensable in a language possessing fewer lexical items than standard English. The varied techniques used to study levels of abstraction in natural categories converged to demonstrate the importance of basic categories. All the studies agreed concerning the level of abstraction at which basic categories were formed.

One major issue which these studies raise is the extent to which structure is "given" by the world versus created by the perceiving organism. Such questions can be discussed on a number of levels. In the first place, the present research is empirical and not intended to be related to any of the classical issues of philosophy. Our claim that there is structure "out there" in the world is not a metaphysical claim about the existence of a world without a knower, but an empirical claim which includes the knower. Given a knower who can perceive the complex attributes of feathers, fur, and wings, it is an empirical fact "out there" that wings co-occur with feathers more than with fur. What kinds of attributes can be perceived is, of course, species specific. A dog's sense of smell is more highly differentiated than a human's and the structure of the world for a dog must surely include attributes of smell which, as a species, we are incapable of perceiving. Furthermore, since a dog's body is constructed differently from a human's, his motor interactions with objects are necessarily differently structured. The "out there" of a bat, a frog, or a bee is undoubtedly more different still than that of a human.

On the empirical level, given that a human is capable of perceiving

as of abstract categories as such. The motor movements of sitting on a chair comprise the function of the chair; however, the hand motions made with respect to a rectangle of paper are not the functions of a negotiable check. The separation of shape and motor movements from the attributes of the object are even more abvious for abstract categories such as causality or democracy. Even for clearly concrete objects, there may be boundary conditions beyond which the present formulation may not be expected to apply. For example, very small objects which have attributes in common and can be used by means of the same motor programs might not necessarily have the similarities in shape which one would expect of larger objects with common attributes and motor programs. 
some set of attributes, and that those attributes possess a correlational structure in the world, the state of knowledge of the person may differ from the potential provided by the world in that: (a) The person may be ignorant of (or indifferent or inattentive to) the attributes, or he may know of the attributes but be ignorant of their correlational structure, and (b) He may know of the attributes and their correlational structure but exaggerate that structure, turning partial into complete correlations (as when attributes true only of many members of a category are thought of as true of all members). Basic objects for an individual, subculture, or culture must result from an interaction between the potential structure provided by the world and the particular emphases and state of knowlege of the people who are categorizing. However, the environment places constraints on categorizations. Human knowledge cannot provide correlational structure where there is none. Humans can only ignore or exaggerate correlational structures.

\section{Ignorance and Knowledge of Existing Structure}

Different amounts of knowledge about objects can change the classification scheme. Thus, experts in some domain of knowledge make use of attributes that are ignored by the average person. The case of airplanes offers a good example. Airplane appeared to be the basic level for most of the students participating in our experiments. One subject, however, was a former airplane mechanic. His taxonomy was interesting. The lists of attributes common to airplanes produced by most subjects were paltry compared to the lengthy lists of additional attributes which he could produce. Furthermore, his motor programs as a mechanic were quite distinct for the attributes of the engines of different types of planes. Finally, his visual view of airplanes was not the canonical top and side images of the public; his canonical view was of the undersides and engines.

We used this subject as an informant (in the anthropological sense). His differentiation of airplanes was not infinite; he considered a single and twin engine Cessna to be quite similar, and he thought that they would probably constitute subordinate categories. Furthermore, he considered airplanes as a whole more similar to each other than different vehicles are to each other. He could take the role of the average person and list attributes common to all airplanes, and could imagine an average airplane shape from the outside.

Thus, categories such as airplanes can have differing sets of correlational structures, depending upon the degree of knowledge of the perceiver. A hypothetical taxonomy of this type and of a potential onelevel type are graphed in Fig. 4. In this figure, basic level objects are depicted as elbows in graphs of common attributes, motor movements, and shapes. 


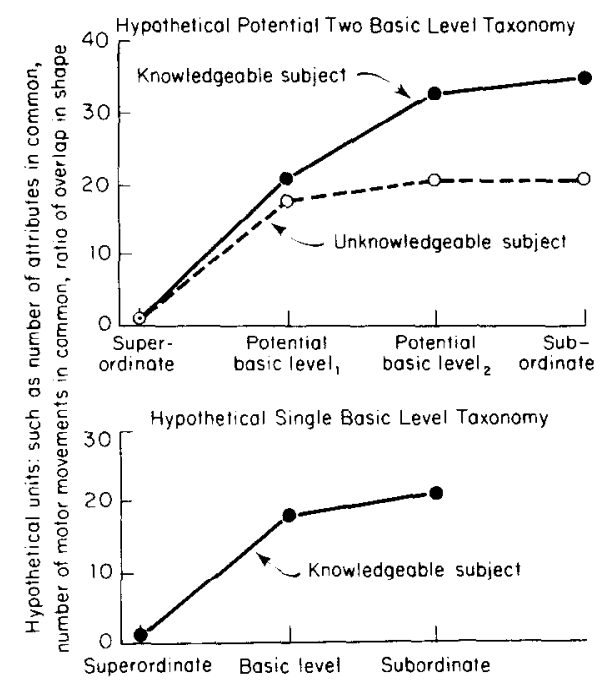

FIG. 4. Hypothetical one- and two-level taxonomies.

We can now discuss the possible reasons for the disparity between our findings with biological taxonomies and those from anthropological and linguistic data (Berlin, 1972). On the one hand, biological taxonomies might actually be of the type in which only one basic level grouping is possible regardless of degree of knowledge, so that the earlier anthropological findings were in error. In support of such a contention is the fact that when one examines the full range of any genus (for example, oak, maple, and birch in The Trees of North America, 1968), one finds that at the genus level, biological classes are not discrete bundles of correlated attributes, but, rather contain many subtypes within the genus whose characteristics merge with those of other genuses. For example, attributes of oaks which one might think would differentiate that genus distinctively from maples - such as tree shape, leaf shape, and seed type - in fact, overlap maples in all of those characteristics when all varieties of the two genuses are considered.

Berlin (Note 4) has countered this argument with the observation that folk biological taxonomies are, by definition, created by the inhabitants of a single ecological zone who come into contact with only a few of the varieties of each genus. Thus, in folk taxonomies, the genus should be a level of abstraction at which potential correlations of attributes exist. That English speakers once made basic level distinctions at the level of the genus is suggested by the number of monolexemic terms for biological genuses available in English (see the bird, fish, and tree names in Battig \& Montague, 1969). Such lists of terms are not similarly available for subordinates of chair, shirt, hammer or for any of the other nonbiological basic level terms in our taxonomies. 
Thus, biological taxonomies are probably of the type in which two basic level groupings are possible, but our city dwelling subjects appeared to be ignorant of the attributes characteristic of the generic level biological categories in their environment.

The effects of expertise on classification requires systematic study. We believe that for all taxonomies there is a level below which further differentiations cannot form basic level categories because, no matter how great the frequency of use of the objects or degree of expertise, there simply is not a sufficient number of attributes to differentiate objects below that level. Thus, for any taxonomy, there should be a level at which the attributes common to objects which are added by further distinctions will be outweighed by the attributes which the newly distinguished classes share with each other. When a further distinction reduces rather than increases the cue validity of categories at the new level of classification, that distinction results in subordinate categories rather than new basic level categories. The only way in which distinctions which are basic level classes can be added indefinitely is to redefine the total set whose structure is at issue to include only the narrowest class under consideration. Thus, Phillips-screwdrivers-1-in.-long and Phillips-screwdrivers-1/2-in.-long can be basic level classes if the total set (the universe of discourse) is defined to include only very short Phillips screwdrivers. While this may be a way to characterize the classification system of a specialized machine, it does not seem to be a reasonable way to characterize the cognitive reorganization which takes place in a human mind with increases in expertise. Both theoretical formulation of the change in knowledge structures resulting from a gain in expert knowledge and empirical studies of the knowledge structures of experts are needed before this question can be discussed intelligently.

One evident aspect of expertise is that the expert's knowledge is probably often confined to specific parts of a taxonomy, thereby, creating unevenness in the expert's categorization of that taxonomy. One can easily imagine a poultry farmer for whom chickens and turkeys (and/or subordinates of these birds) are basic level objects but for whom the rest of the bird class remains undifferentiated. One can imagine an antique furniture dealer for whom Chippendale and Hepplewait chairs are basic level objects, but for whom kitchen and living room chairs, in the average house, are as undifferentiated as for our subjects. Indeed, differentiation of mammals (but not birds, fish, and other major divisions of animals) into basic level objects can be observed in our own culture which is more knowledgeable about mammals than other animal classes. This may be why mammal is infrequently used by English speakers; mammals are thought of as members of their basic level classes and are called by their basic names. The names of mammals are one level in the taxonomy lower than are the basic level classes and names for other major animal classifications. 


\section{Exaggeration of Structure: Prototypes}

Any person or culture may exaggerate existing structure so that attributes, motor movements, and shapes characteristic of only some members of a category may be thought of as though they were characteristic of all. By such a mechanism, the basic category cuts in the world are made to appear even simpler and more structured than they are in reality. Such simplification may occur through the coding of categories in cognition in terms of prototypes of the most characteristic members of the category. Thus, even when correlational structure in the world is only partial, or when attributes are continuous, categories can be maintained as discrete by their mode of cognitive coding.

At this point, we can comment on the relationship between the present research on the taxonomic structure of categories and earlier research on the internal structure of categories and coding in terms of prototypes. There is considerable evidence that some natural categories are continuous rather than definitively bounded entities (see Lakoff, 1972; Rosch, 1973; Zadeh, 1965). Some natural, continuous categories seem to be structured cognitively into items which differ in their degree of prototypicality - that is, in the degree to which the items match clear clases or good examples of the category (see Rosch, in press, for summary of evidence).

Rosch and Mervis (1975) have shown that the more an item is judged to be prototypical of a category, the more attributes it has in common with other members of the category and the fewer attributes in common with members of contrasting categories. This finding was demonstrated for natural language superordinate categories, for natural language basic level categories, and for artificial categories in which the amount of experience with the items was controlled. Thus, prototypes of categories appear to follow the same principles as basic categories. Prototypes appear to be just those members of a category which most reflect the redundancy structure of the category as a whole. Categories form to maximize the information-rich clusters of attributes in the environment and, thus, the cue validity of the attributes of categories. Prototypes of categories appear to form in such a manner as to maximize the clusters and cue validity within categories.

Given these assumptions, we can now interpret some apparently anomolous data. In Experiment 1, the attributes listed by subjects and verified as true of the category as a whole by the judges, were not true for every example conceivable of the category. A reasonable explanation for this finding is that subjects and judges were thinking of prototypical category members when making the judgments: Attributes that apply to prototypical members need not be true of all items classifiable as members of the category. The experiments of Rosch and Mervis (1975) 
support this view. They asked subjects to list attributes for 20 members of varying prototypicality from each of six superordinate categories. There were virtually no attributes common to all members of these superordinate categories. However, the five items rated most prototypical within each category tended to have many attributes in common with each other.

The exaggeration of category structure contained in the prototype makes prototypes potentially useful in cognitive processes. Matching to a prototype in categorization would allow humans to make use of their knowledge of the contingency structure of the environment without the laborious process of computing and summing the validities of individual cues (Rosch \& Mervis, 1975). The imageability of prototypes of basic level categories may provide additional advantages (Paivio, 1971).

Just as selective ignorance and expertise about categories create unevenness in the categorizations within a taxonomy, variation in basic level classes and coding in terms of prototypes undoubtedly creates unevenness in classifications within basic level categories. Objects nominally within a basic level class may be sufficiently divergent from typical members of the class that they may constitute a separate potential or actual basic level category. For example, consider the objects called bean bag chairs in English. These may have attributes, motor movements, and shapes in common with each other and sufficiently different from those of typical chairs that bean bag chairs are thought of as a basic level category separate from and on the same level of abstraction as chair. The same is very probably the case for atypical members of biological categories. Although bird, fish, and tree were basic level classes for our subjects, members of these classes such as penguin, lobster, and palm tree, undoubtedly constitute classes differentiated from the basic level categories as a whole. Thus, while a typical looking chair or bird may be perceived and recognized first as a member of the basic level class chair or bird, a sufficiently deviant member is probably perceived first as a member of its individual class (e.g., bean bag chair, flamingo). One obvious implication of this is that deviant items should be verified faster for their "subordinate" name than for the name of the basic level category to which they are allied. Other implications concern the name that will be used in a free naming situation such as that in Experiment 10 and the name first learned by children.

\section{Universality of the Principles of Categorization}

From the beginning of the present paper, it has been implied that this is a study of universal principles of categorization. But what aspects of the theory are intended to be universals? The content of categories should not be. It has been argued that categories reflect both real world corre- 
lational structure and the state of knowledge of that structure of the people doing the categorizing. Since the structure of the environment differs greatly in different parts of the world, one expects the categories of different cultures to differ. In addition, interest in and knowledge of attributes and their correlation for specific domains differ among cultures, subcultures, and individuals.

It is the principle of category formation that is claimed to be universal. On the most general level, categories form so as to be maximally differentiable from each other. This is accomplished by categories which have maximum cue validity-i.e., categories that have the most attributes common to members of the category and the least attributes shared with members of other categories. While specific principles such as common motor movements and shapes apply only to concrete objects, this most general principle may be applicable to other domains as well. For example, the principle is similar to a recent account of how visual figures are segmented into parts (Palmer, 1975), and it may provide insights into how the stream of experience becomes segmented into events.

In summary: The correlational structure of the environment, modified by selective ignorance and exaggeration of the attributes and structure of that environment, are mirrored in categorization systems. Segmentation of experience occurs to form basic levels which maximize the differentiability of categories. For categories of concrete objects, basic objects are the most general classes at which attributes are predictable, objects of the class are used in the same way, objects can be readily identified by shape, and at which classes can be imaged. Basic objects should generally be the most useful level of classification. Universally, basic object categories should be the basic classifications made during perception, the first learned and first named by children, and the most codable, most coded, and most necessary in the language of any people.

\section{APPENDIX 1 \\ Examples of Judge-Amended Attribute Lists}

Note: Lower levels are assumed to include all attributes listed at higher levels; only attributes new to a lower level are listed.

$\begin{array}{llll}\text { Tool } & \text { Clothing } & \text { Furniture } & \text { Bird } \\ \text { make things } & \text { you wear it } & \text { no attributes } & \text { feathers } \\ \text { fix things } & \text { keeps you warm } & \text { Chair } & \text { wings } \\ \text { metal } & \text { Pants } & \text { legs } & \text { beak } \\ \text { Saw } & \text { legs } & \text { seat } & \text { legs } \\ \text { handle } & \text { buttons } & \text { back } & \text { feet } \\ \text { teeth } & \text { belt loops } & \text { arms } & \text { eyes }\end{array}$


APPENDIX I (continued)

$\begin{array}{ll}\begin{array}{l}\text { Tool } \\ \text { blade }\end{array} & \begin{array}{l}\text { Clothing } \\ \text { pockets }\end{array} \\ \text { sharp } & \text { cloth } \\ \text { cuts } & \text { two legs } \\ \text { edge } & \text { Levis } \\ \text { wooden handle } & \text { blue } \\ \text { Cross-cutting } & \text { Double knit } \\ \quad \text { hand saw } & \text { pants } \\ \text { used in } & \text { comfortable } \\ \quad \text { construction } & \text { stretchy } \\ \text { Hack hand saw } & \\ \text { no additional } & \end{array}$

Furniture
comfortable
four legs
wood
holds people--
$\quad$ you sit on it
Kitchen chair
no additional
Living room chair
large
soft
cushion

Bird

tail

head claws

lays eggs nests

flies

chirps

eats worms and flies

Sparrow

small

brown

Song sparrow no additional

Field sparrow no additional

\section{APPENDIX II}

\section{Examples of Motor Movement Sequences}

Note: Movements tallied for subordinate classes are the same ones listed in this appendix for the basic level unless otherwise indicated.

" " " indicates a movement which was tallied for the subordinate class that had not been listed for the basic level of that class.

" -" indicates a movement which failed to be tallied for the subordinate class which had been listed for the basic level of that class.

\begin{tabular}{|c|c|c|c|}
\hline Tool & Clothing & Furniture & Bird (look at) \\
\hline Hand: grasp & Eyes: scan & Eyes: scan & Eyes: scan \\
\hline Fingers: grasp & Hand: grasp & Chair (sit on) & pursue \\
\hline Hammer & Pants (put on) & Head: turn & look up \\
\hline Arm: extend & Hands: grasp & Body: turn & squint \\
\hline Hand: big grasp & Arms: extend & move & $\begin{array}{r}\text { blink } \\
\text { Head. turn }\end{array}$ \\
\hline $\begin{array}{l}\text { Fingers: } \\
\text { position }\end{array}$ & $\begin{array}{l}\text { Feet: position } \\
\text { Knee: bend }\end{array}$ & $\begin{array}{l}\text { position } \\
\text { Knees: bend }\end{array}$ & $\begin{array}{c}\text { pursue } \\
\text { Neck: tip back }\end{array}$ \\
\hline $\begin{array}{l}\text { Other hand: } \\
\text { position }\end{array}$ & $\begin{array}{l}\text { Leg: raise } \\
\quad \text { extend }\end{array}$ & $\begin{array}{l}\text { Arm: extend- } \\
\text { touch }\end{array}$ & Sparrow \\
\hline $\begin{array}{l}\text { Body: bend } \\
\text { Neck: bend }\end{array}$ & $\begin{array}{c}\text { Foot: raise } \\
\text { extend }\end{array}$ & $\begin{array}{l}\text { Waist: bend } \\
\text { Butt: touch }\end{array}$ & $\begin{array}{l}\text { Eyes: scan } \\
\text { pursue }\end{array}$ \\
\hline
\end{tabular}


APPENDIX II (continued)

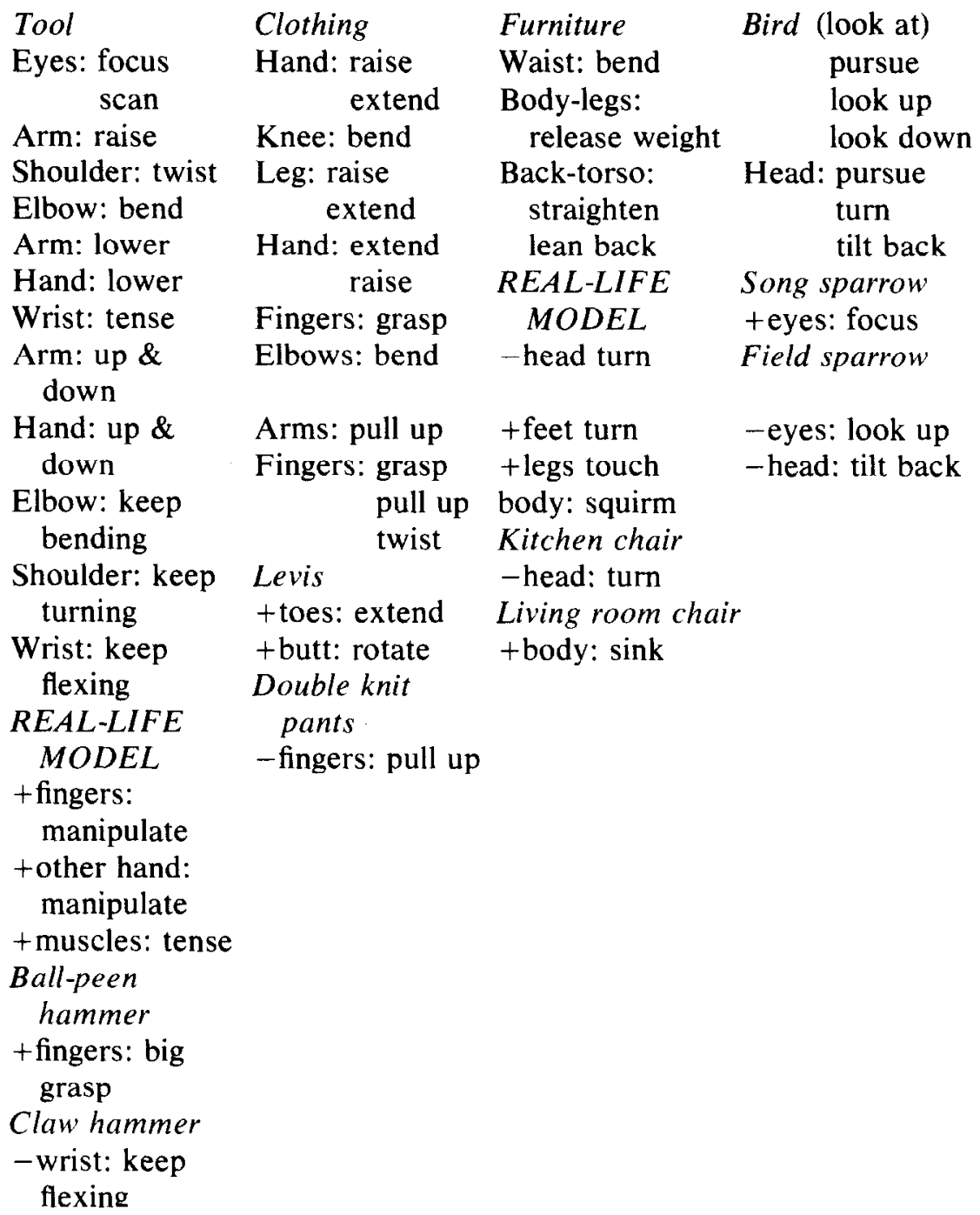

REFERENCES

Anglin, J. The child's first terms of reference. In S. Ehrlich et E. Tulving (Eds.) La Memoire Semantique. Paris: Bulletin de Psychologie, 1976.

Annett, M. The classification of instances of four common class concepts by children and adults. British Journal of Educational Psychology, 1959, 29, 223-236.

Attneave, F., \& Arnoult, M. D. The quantitative study of shape and pattern perception. Psychological Bulletin 1956, 53, 452-471.

Battig. W. F., \& Montague. W. E. Category norms for verbal items in 56 categories: A replication and extension of the Connecticut category norms. Journal of Experimental Psychology, 1969, 80 (Monograph Supplement 3, Part 2). 
Beach, L. R. Cue probabilism and inference behavior. Psychological Monographs, 1964, 78, Whole No. 582. (a)

Beach, L. R. Recognition, assimilation, and identification of objects. Psychological Monographs, 1964, 78, Whole No. 583. (b)

Beller, H. K. Priming: Effects of advance information on matching. Journal of Experimental Psychology 1971, 87, 176-182.

Berlin, B. Speculations on the growth of ethnobotanical nomenclature. Language in Society 1972, 1, 51-86.

Berlin, B., Breedlove, D. E., \& Raven, P. H. Folk taxonomies and biological classification. Science 1966, 154, 273-275.

Berlin, B., Breedlove, D. E., \& Raven, P. H. General principles of classification and nomenclature in folk biology. American Anthropologist, 1973, 75, 214-242.

Birds of North America: A guide to field identification. New York: Golden Press, 1966.

Brown, R. A first language. Cambridge, MA: Harvard University Press, 1974.

Bruner, J. S., Olver, R. R., \& Greenfield, P. M. Studies in cognitive growth. New York: Wiley, 1966.

Brunswik, E. Perception and the representative design of experiments. Berkeley: University of California Press, 1956.

Bulmer, R. Why is the cassowary not a bird? A problem of zoological taxonomy among the Karam of the New Guinea Highlands. Man: the Journal of the Royal Anthropological Institute, 1967, 2, 5-25.

Bulmer, R., \& Tyler, M. J. Karam classification of frogs. The Journal of the Polynesian Society, 1968, 77, 333-385.

Clark, H. H. The language-as-fixed-effect fallacy: A critique of language statistics in psychological research. Journal of Verbal Learning and Verbal Behavior, 1973, 12, 335-359.

Color treasury of acquarium fish. New York: Crcscent Books, 1972.

Denney, N. W. Evidence for developmental changes in categorization criteria for children and adults. Human Development, 1974, 17, 41-53.

Frake, C. O. The ethnographic study of cognitive systems. In S. A. Tyler (Ed.), Cognitive anthropology. New York: Holt, Rinehart \& Winston, 1969.

Garner, W. R. The processing of information and structure. New York: Wiley, 1974.

Garrettson, J. Cognitive style and classification. Journal of Genetic Psychology, 1971, 119, 79-87.

Gelman, R. The nature and development of early number concepts. In H. Reese (Ed.), Advances in child development and behavior. New York: Academic Press, 1972, Vol. 7.

Goldberg, S., Perlmutter, M., \& Myers, W. Recall of related and unrelated lists by 2year-olds. Journal of Experimental Child Psychology, 1974, 18, 1-8.

The golden book of bird stamps. New York: Golden Press, 1966.

Goldman, A. E., \& Levine, M. A developmental study of object-sorting. Child Development, 1963, 34, 649-666.

Kahneman, D., \& Tversky, A. On the psychology of prediction. Psychological Review, $1973,80,237-251$.

Kay, P. Taxonomy and semantic contrast. Language, 1971, 47, 866-887.

Klima, E. S., \& Bellugi, U. Perception and production in a visually based language. In D. Aaronson \& R. W. Rieber (Eds.) Developmental psycholinguistics and communication disorders. New York: New York Academy of Sciences, 1975.

Kucera, H., \& Francis, W. N. Computational analysis of present-day American English. Providence: Brown University Press, 1967.

Lakoff, G. Hedges: A study in meaning criteria and the logic of fuzzy concepts. Papers from the Eighth Regional Meeting, Chicago Linguistics Society, Chicago: University of Chicago Linguistics Department, 1972. 
Leach, E. Anthropological aspects of language: Animal categories and verbal abuse. In E. H. Lenneberg (Ed.), New directions in the study of language. Cambridge, MA: MIT Press, 1964.

Paivio, A. Imagery and verbal processes. New York: Holt, Rinehart \& Winston, 1971.

Palmer, S. E. Visual perception and world knowledge. In D. A. Norman, D. E. Rumelhart, \& the LNR Research Group. Explorations in cognition. San Francisco: W. H. Freeman, 1975.

Peterson, M. J., \& Graham, S. E. Visual detection and visual imagery. Journal of Experimental Psychology, 1974, 103, 509-514.

Piaget, J. The origins of intelligence in children. New York: International Universities Press, 1952.

Reed, S. K. Pattern recognition and categorization. Cognitive Psychology, 1972, 3, 382-407.

Rosaldo, M. Z. Metaphors and folk classification. Southwestern Journal of Anthropology, 1972, 28, 83-99.

Rosch, E. On the internal structure of perceptual and semantic categories. In T. E. Moore (Ed.), Cognitive development and the acquisition of language. New York: Academic Press, 1973.

Rosch, E. Cognitive representations of semantic categories. Journal of Experimental Psychology: General, 1975, 104, 192-233. (a)

Rusch, E. The nature of mental codes for color categories. Journal of Experimental Psychology: Human Perception and Performance, 1975, 1, 303-322. (b)

Rosch, E. Human categorization. In N. Warren (Ed.), Advances in cross-cultural psychology. (Vol. 1). London: Academic Press, in press.

Rosch, E., \& Mervis, C. B. Family resemblances: Studies in the internal structure of categories. Cognitive Psychology, 1975, 7, 573-605.

Segal, S. J. Processing of the stimulus in imagery and perception. In S. J. Segal (Ed.), Imagery. New York: Academic Press, 1971.

Segal, S. J., \& Fusella, V. Influence of imaged pictures and sounds on detection of auditory and visual signals. Journal of Experimental Psychology, 1970, 83, 458-464.

Thompson, J. The ability of children of different grade levels to generalize on sorting tests. Journal of Psychology, 1941, 11, 119-126.

The trees of North America: A guide to field identification. New York: The Golden Press, 1968.

Tversky, A., \& Kahneman, D. Availability: A heuristic for judging frequency and probability. Cognitive Psychology, 1973, 5, 207-232.

Vygotsky, L. S. Thought and language. New York: Wiley, 1962.

Zadeh, L. A. Fuzzy sets. Information and Control, 1965, 8, 338-353.

\section{REFERENCE NOTES}

1. Rosch, E., Mervis, C. B., Gray, W., Johnson, D., \& Boyes-Braem, P. Basic objects in natural categories. Working Paper No. 43. The Language Behavior Research Laboratory, University of California, Berkeley, California 94720.

2. Nash, S. C. The use of free recall to infer cognitive organization in three, four, and five year olds. Unpublished MA thesis, University of Pennsylvania, 1973.

3. Carey, S., DeVilliers, J., \& DeVilliers, P. A new method for analyzing children's concepts. Cases 1 and 2: The child's concept of life and the child's concept of animal. Final report for NIMH Grant No. MH24922, 1975.

4. Berlin, B. Personal communication, March 1974. 